\title{
Evaluation of Large-Eddy Simulations via Observations of Nocturnal Marine Stratocumulus
}

\author{
Bjorn Stevens, ${ }^{a}$ Chin-Hoh Moeng, ${ }^{\mathrm{b}}$ Andrew S. Ackerman, ${ }^{\mathrm{c}}$ Christopher S. Bretherton, ${ }^{\mathrm{d}}$ \\ Andreas Chlond, ${ }^{\mathrm{e}}$ Stephan de Roode, ${ }^{\mathrm{f}}$ James Edwards, ${ }^{\mathrm{g}}$ Jean-Christophe Golaz, ${ }^{\mathrm{h}}$ Hongli Jiang, ${ }^{\mathrm{i}}$ \\ Marat Khairoutdinov, ${ }^{i}$ Michael P. Kirkpatrick, ${ }^{j}$ David C. Lewellen, ${ }^{k}$ Adrian Lock, ${ }^{1}$ \\ Frank Müller, ${ }^{\mathrm{e}}$ David E. Stevens, ${ }^{\mathrm{m}}$ EoIn Whelan, ${ }^{1}$ And Ping Zhu ${ }^{\mathrm{d}}$ \\ ${ }^{a}$ Department of Atmospheric and Oceanic Sciences, University of California, Los Angeles, Los Angeles, California \\ ${ }^{b}$ National Center for Atmospheric Research, Boulder, Colorado \\ ${ }^{c} N A S A$ Ames Research Center, Moffett Field, California \\ ${ }^{d}$ Department of Atmospheric Sciences, University of Washington, Seattle, Washington \\ ${ }^{e}$ Max Planck Institute for Meteorology, Hamburg, Germany \\ ${ }^{f}$ Institute for Marine and Atmospheric Research, Utrecht, Netherlands \\ ${ }^{g}$ IBM Business Consulting Services, Boulder, Colorado \\ ${ }^{h}$ National Research Council, Naval Research Laboratory, Monterey, California \\ ${ }^{i}$ Department of Atmospheric Science, Colorado State University, Fort Collins, Colorado \\ ${ }^{j}$ School of Engineering, University of Tasmania, Tasmania, Australia \\ ${ }^{k}$ MAE Department, West Virginia University, Morgantown, West Virginia \\ ${ }^{l}$ Met Office, Exeter, Devon, United Kingdom \\ ${ }^{m}$ Lawrence Livermore National Laboratory, Livermore, California
}

(Manuscript received 15 September 2004, in final form 5 November 2004)

\begin{abstract}
Data from the first research flight (RF01) of the second Dynamics and Chemistry of Marine Stratocumulus (DYCOMS-II) field study are used to evaluate the fidelity with which large-eddy simulations (LESs) can represent the turbulent structure of stratocumulus-topped boundary layers. The initial data and forcings for this case placed it in an interesting part of parameter space, near the boundary where cloud-top mixing is thought to render the cloud layer unstable on the one hand, or tending toward a decoupled structure on the other hand. The basis of this evaluation consists of sixteen 4-h simulations from 10 modeling centers over grids whose vertical spacing was $5 \mathrm{~m}$ at the cloud-top interface and whose horizontal spacing was $35 \mathrm{~m}$. Extensive sensitivity studies of both the configuration of the case and the numerical setup also enhanced the analysis. Overall it was found that (i) if efforts are made to reduce spurious mixing at cloud top, either by refining the vertical grid or limiting the effects of the subgrid model in this region, then the observed turbulent and thermodynamic structure of the layer can be reproduced with some fidelity; (ii) the base, or native configuration of most simulations greatly overestimated mixing at cloud top, tending toward a decoupled layer in which cloud liquid water path and turbulent intensities were grossly underestimated; (iii) the sensitivity of the simulations to the representation of mixing at cloud top is, to a certain extent, amplified by particulars of this case. Overall the results suggest that the use of LESs to map out the behavior of the stratocumulus-topped boundary layer in this interesting region of parameter space requires a more compelling representation of processes at cloud top. In the absence of significant leaps in the understanding of subgrid-scale (SGS) physics, such a representation can only be achieved by a significant refinement in resolution-a refinement that, while conceivable given existing resources, is probably still beyond the reach of most centers.
\end{abstract}

\section{Introduction}

Large-eddy simulation (LES) has profoundly impacted the way we study the planetary boundary layer

Corresponding author address: Bjorn Stevens, Department of Atmospheric and Oceanic Sciences, University of California, Los Angeles, 405 Hilgard Ave., Box 951565, Los Angeles, CA 900951565.

E-mail: bstevens@atmos.ucla.edu
(PBL). PBLs, particularly those involving clouds, are impossible to represent with fidelity in the laboratory, and very difficult and expensive to probe observationally. In such cases LES is often used as a basis for developing our intuition and guiding our theories. This special status of LES, as a proxy for reality, stems partly from its similitude to real flows, but also because the methodology is generally thought to be insensitive to formulaic details and arbitrary parameters. Both quali- 
ties follow because the method resolves the energetics of the large eddies, which are responsible for most of the transport of momentum, energy, and mass through the bulk of the PBL. More quantitative evaluations of the method are scarce for precisely the reason that LES has become so popular-real flows are challenging to quantify. More than 30 years ago Bradshaw (1972) coined the phrase "fact gap" to describe this state of affairs, a gap which some (e.g., Wyngaard 1998) believe is widening.

In the absence of decisive measurements the principal method of evaluating LES has been through exhaustive sensitivity studies of individual models and comparisons among many models. The hope is that the robustness of the method testifies to its fidelity. For the most part such studies have cast a favorable light on LES. However, a couple of studies (e.g., Moeng et al. 1996; Stevens et al. 2001) have raised serious questions about the ability of LES to realistically represent cloudtopped PBLs capped by a strong temperature inversion. In a study by Moeng et al. (1996), 12 large-eddy simulation codes were run for identical initial conditions and forcings loosely based on a cloud layer observed during the First International Satellite Cloud Climatology Project (ISCCP) regional field study (Albrecht et al. 1988). An evaluation of the simulations revealed marked differences, with global integrals of the flow, such as liquid water path, differing by as much as an order of magnitude. The origin of these differences was conjectured to be changes in the degree to which different models entrained warm and dry air from the overlying free troposphere into the turbulent PBL. But this idea was difficult to test because of the confounding effects from different representations of important physical processes, for example, radiative transfer and thermodynamic and cloud microphysical processes.

To help isolate the origin of these sensitivities, a much more idealized test case was formulated, the socalled smoke cloud (Lilly 1968; Bretherton et al. 1999), wherein a neutrally stratified mixed layer is filled with radiatively opaque smoke and capped by a strong jump in density (temperature). The more dense lower layer is driven by the radiative cooling (prescribed by a simple formula that depends on the smoke path) from the top of the smoke layer. The ensuing turbulence is then thought to be responsible for any entrainment (mixing) of much warmer, smokeless, free-tropospheric air into the turbulent layer. Because the amount of entrainment is not externally constrained, this type of test case isolates the central question of how efficiently the simulated layer entrains. Given the simplicity of the formulation, differences could be readily attributed to either the numerical implementation of the basic equations being solved, or the model used to represent unre- solved scales of motion. Results from this study showed that for poor vertical resolution, simulations differed markedly in their resultant entrainment rates, but as the vertical grid was refined, entrainment rates were reduced and the scatter amongst models and their degree of disagreement with laboratory analogs to the smoke cloud also appeared to be reduced (Bretherton et al. 1999).

Based on the smoke cloud results a consensus emerged that very fine vertical resolution (order $5 \mathrm{~m}$ ) was necessary to properly capture entrainment in stratocumulus-like, radiatively driven, flows. Most studies showed markedly less sensitivity to horizontal resolution [and by implication grid anisotropy (Lewellen and Lewellen 1998; Stevens et al. 1999)]. Because for coarse horizontal resolution the only scales that were being well represented were those commensurate with the largest eddies, these results led to the view that the energetics of the PBL-scale eddies determines the rate of entrainment, irrespective of how the entrainment process itself is captured by the simulation. This thinking helped rationalize a number of studies (e.g., Lewellen and Lewellen 1998; Lock 1998; vanZanten et al. 1999; Moeng 2000) that then independently revisited the more complex stratocumulus problem with an eye toward mapping out how entrainment rates depend on flow parameters-this being the central question for more aggregated models of PBL flows.

It soon became apparent that simulations by different LES models led to markedly different entrainment parameterizations (Stevens 2002). Even for fine vertical resolution, overall entrainment efficiencies from different codes appeared to differ by a factor of 2 or more, suggesting that the sensitivities to first emerge in the intercomparison study of Moeng et al. (1996) were not completely resolved by using fine vertical resolution. For such large disparities it became possible to imagine (cf. Lenschow 1996) collecting data to help resolve the differences among models, which in large part motivated the second Dynamics and Chemistry of Marine Stratocumulus (DYCOMS-II) field study (Stevens et al. 2003b). By focusing the observations on nocturnal stratocumulus, for which the forcings tend to be more stationary and easy to characterize, one could hope to close the energy budget for the cloud layer. By using a variety of tracers, including dimethyl sulfide [DMS, whose qualities are particularly well suited to measuring entrainment rates Lenschow (1996)], it was possible to estimate entrainment rates to within $30 \%-$ narrowing, if you will, Bradshaw's fact gap.

In the current study we use data from the first research flight (RF01) of DYCOMS-II (Stevens et al. 
2003a) and return to the intercomparison framework of Moeng et al. (1996) to reevaluate large-eddy simulation of stratocumulus-topped PBLs. We return to the intercomparison framework for a number of reasons: (i) it touches base with our earlier point of departure (e.g., Moeng et al. 1996); (ii) it provides a compelling framework for the evaluation of PBL parameterizations as manifest in single-column models [e.g., see the accompanying manuscript by Zhu et al. (2005 hereafter ZHU)]; (iii) it helps highlight the extent to which one of the underpinnings of LES, namely its supposed insensitivity to formulation details, is true, at least for the climatologically critical flow regime of marine stratocumulus. A critical distinction between this and previous intercomparison studies is the extent to which we know the right answer. Such a distinction is also critical to the use of the LES results such as ours to help inform the development and evaluation of PBL parameterizations as is discussed in an accompanying manuscript by ZHU.

\section{Methodology}

\section{a. The case: DYCOMS-II, RF01}

Of the seven nocturnal flights flown during DYCOMSII, the first (RF01) shall serve as the basis for our study. RF01 had a number of appealing features that make it well suited for a study of this type: The environmental conditions were relatively homogeneous; the cloud layer persisted despite the presence of mean conditions that, according to some theories (e.g., Randall 1980; Deardorff 1980a), should have led to its dissipation; it was not complicated by the presence of significant drizzle; its energetics appeared robust to a variety of observational analyses (cf. Stevens et al. 2003a; Faloona et al. 2005; Gerber et al. 2005); and preliminary attempts to simulate it were encouraging.

A detailed description of RF01, the methods used to observe it, and their associated uncertainties, is provided by Stevens et al. (2003a). For the purposes of this study we here limit ourselves to summarizing its main features as used to initialize and force the LES. These initial conditions and forcings differ from those used in the previous study principally through the incorporation of a more realistic representation of the temperature structure of the free atmosphere and the processes (radiation and large-scale subsidence) that help determine it.

\section{1) Mean state}

The basic state for RF01 was compiled from all of the measurements and is idealized as a quasi-two-layer structure in liquid water potential temperature $\theta_{l}$ and total-water specific humidity $q_{t}$ according to

$$
\begin{aligned}
\theta_{l} & = \begin{cases}289.0 \mathrm{~K} & z \leq z_{i} \\
297.5+\left(z-z_{i}\right)^{1 / 3} \mathrm{~K} & z>z_{i},\end{cases} \\
q_{t} & = \begin{cases}9.0 \mathrm{~g} \mathrm{~kg}^{-1} & z \leq z_{i} \\
1.5 \mathrm{~g} \mathrm{~kg}^{-1} & z>z_{i},\end{cases}
\end{aligned}
$$

where $z_{i}$ is initially set to $840 \mathrm{~m}$, and $\theta_{l}$ was derived from the measured air temperature using values of the physical constants: $c_{p}=1.015 \mathrm{~kJ} \mathrm{~kg}^{-1} \mathrm{~K}^{-1}, R_{d}=0.287 \mathrm{~kJ}$ $\mathrm{kg}^{-1} \mathrm{~K}^{-1}$, and $L_{v}=2.47 \mathrm{MJ} \mathrm{kg}^{-1}$, and a surface pressure of $1017.8 \mathrm{hPa} .{ }^{1}$ To better match the profile for $\theta_{l}$ to the observations in the free troposphere, its values above $z_{i}$ are reduced by $1.5 \mathrm{~K}$ as compared to what was used in Stevens et al. (2003a).

Other required forcings include geostrophic winds, divergence, sea surface temperatures, and radiation. We specify geostrophic winds of $U_{g}=7 \mathrm{~m} \mathrm{~s}^{-1}$ and $V_{g}=$ $-5.5 \mathrm{~m} \mathrm{~s}^{-1}$, which produce winds within the PBL near 6 and $-4.25 \mathrm{~m} \mathrm{~s}^{-1}$, respectively, as observed. The divergence of the large-scale winds is taken to be $D=$ $3.75 \times 10^{-6} \mathrm{~s}^{-1}$ as this seems most consistent with the observed temperature structure above the PBL, and the calculated radiative forcing (see below). For the sea surface temperature we specify a value of $292.5 \mathrm{~K}$, which is $2.1 \mathrm{~K}$ warmer than the surface air temperature. Given a bulk aerodynamic drag coefficient, $C_{D}=C_{H}=$ $C_{Q}=0.0011$, this should correspond to a surface sensible heat flux near $15 \mathrm{~W} \mathrm{~m}^{-2}$ and a surface latent heat flux of approximately $115 \mathrm{~W} \mathrm{~m}^{-2}$. To test the degree to which the assumption of fixed fluxes masked differences among the simulations, additional simulations were performed by most groups for which surface temperatures were held fixed and surface fluxes were computed interactively. However, these simulations did not differ substantially from those with surface fluxes fixed at the above values, and so the results reported on below are from simulations with specified fluxes. The surface temperature and pressure correspond to a surface air density, $\rho_{0}=1.22 \mathrm{~kg} \mathrm{~m}^{-3}$ and an air density just below cloud top of $\rho_{i}=1.13 \mathrm{~kg} \mathrm{~m}^{-3}$, both of which are necessary to convert the energetic fluxes into kinematic fluxes for use in Boussinesq models that set their density to some fixed value with height.

\section{2) RADiATIVE FORCING}

To parameterize the radiative forcing we started with detailed calculations using the $\delta$-four stream radiative-

\footnotetext{
${ }^{1}$ Note that $c_{p}$ can vary substantially across the cloud-top interface: for dry air $c_{\mathrm{pd}}=1005 \mathrm{~J} \mathrm{~kg}^{-1} \mathrm{~K}^{-1}$, while for water vapor $c_{\mathrm{p} v}$ $=1870 \mathrm{~J} \mathrm{~kg}^{-1} \mathrm{~K}^{-1}$, which implies that the isobaric specific heat above the inversion is approximately $1008 \mathrm{~J} \mathrm{~kg}^{-1} \mathrm{~K}^{-1}$ and $1022 \mathrm{~J}$ $\mathrm{kg}^{-1} \mathrm{~K}^{-1}$ below, hence our use of an intermediate value that excludes a dependence on the amount of ambient water vapor.
} 


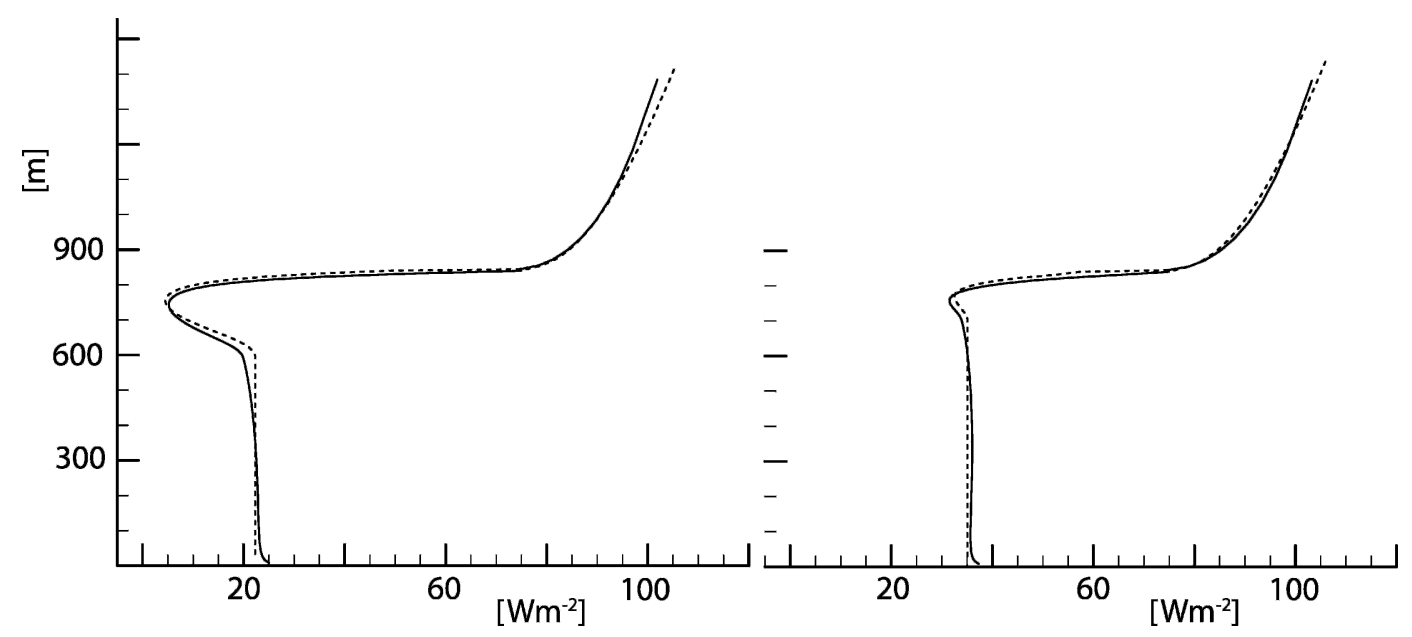

FIG. 1. Idealized net longwave radiative flux from the $\delta$-four stream (solid) and from Eq. (3) (dashed) for (left) the base case and (right) a drier PBL with a thinner cloud. Parameter values used in Eq. (3) are given in the text.

transfer code developed by Fu and Liou (1993). The radiative fluxes from this model were computed given the observed state matched to a free-atmospheric sounding as discussed by Stevens et al. (2003a). Based on the more exact radiative flux profiles derived from the $\delta$-four stream model we constructed a simple model of the net longwave radiative flux as

$$
\begin{aligned}
F_{\mathrm{rad}}(x, y, z, t)= & F_{0} e^{-Q(z, \infty)}+F_{1} e^{-Q(0, z)} \\
& +\rho_{i} c_{p} D \alpha_{z}\left[\frac{\left(z-z_{i}\right)^{4 / 3}}{4}+z_{i}\left(z-z_{i}\right)^{1 / 3}\right],
\end{aligned}
$$

where

$$
Q(a, b)=\kappa \int_{a}^{b} \rho r_{l} d z .
$$

The three terms of (3) separately represent the effects of cloud-top cooling, cloud-base warming, and cooling in the free troposphere just above cloud top, with tuning parameters $\alpha_{z}, \kappa, F_{0}$, and $F_{1}$. The third term in (3) was chosen so that it generates the observed $\left(z-z_{i}\right)^{1 / 3}$ structure in the $\theta_{l}$ profile for $z>z_{i}$ given the large-scale subsidence velocity $W=-D z$. It depends on a determination of $z_{i}$, which in the simulations is identified with the local position of the $8.0 \mathrm{~g} \mathrm{~kg}^{-1}$ isoline of $q_{t}$. The dependence of $F_{\text {rad }}$ on $x, y$, and $t$ is imparted by the spatiotemporal variability in both $r_{l}$ and $z_{i}$.

Figure 1 shows that for the chosen parameter values of $F_{0}=70 \mathrm{~W} \mathrm{~m}^{-2}, F_{1}=22 \mathrm{~W} \mathrm{~m}^{-2}, \kappa=85 \mathrm{~m}^{2} \mathrm{~kg}^{-1}, \alpha_{z}$ $=1 \mathrm{~m}^{-4 / 3}$, and $D=3.75 \times 10^{-6} \mathrm{~s}^{-1}$ the model gives a good fit to the $\delta$-four stream results. We show the fit for two cases, one being the control case with the specified cloud layer, the other being for a drier PBL $\left(q_{t}=8.5\right.$ $\left.\mathrm{g} \mathrm{kg}^{-1}\right)$ and hence a much thinner cloud $\left(q_{l, \text { max }}=0.25\right.$ $\mathrm{g} \mathrm{kg}^{-1}$ ). Not only does (3) well represent the radiative fluxes as simulated by the $\delta$-four stream model, it also appears to capture the basic nature of the sensitivity of these fluxes to significant changes in the state of the cloud layer.

This parameterization of the radiative fluxes was chosen primarily for ease of comparison. It naturally raises a number of subsidiary questions. For instance, to what extent are the details of the radiative forcing important for cloud evolution, particularly the flux divergence in the clear air above the cloud layer and the warming near cloud base. To investigate some of these issues groups were also asked to submit simulations of two additional cases, one which included only the radiative forcing across cloud top, that is, the first term in (3) but with $F_{0}=48 \mathrm{~W} \mathrm{~m}^{-1}$, which maintained the net forcing across the PBL as a whole, and a second case that only omitted the third term in (3). For the most part simulations did not appear to differ substantially from these changes in the forcings; however, we maintained the more realistic parameterization as given by (3) because it better maintained the thermal structure in the free troposphere.

\section{b. Simulation codes and configuration}

Ten different modeling centers participated in the intercomparison, including most of the original participants in the Moeng et al. (1996) study. In addition to a number of standard LES models that enjoy wide use around the world [e.g., National Center for Atmospheric Research (NCAR) and the Met Office (METO)] the ensemble includes two mesoscale models [e.g., the Coupled Ocean-Atmosphere Mesoscale Prediction System (COAMPS) and Regional Atmospheric Modeling System (RAMS)] configured to behave like LES. Detailed descriptions of the participating models 
are provided in appendix A; they differed primarily in their choice of numerical algorithms and in their representation of unresolved motions.

Each participating group was asked to perform a 4-h simulation on a horizontal grid of 96 by 96 points with a 35-m spacing between grid nodes. Vertical spacings were required to be $5 \mathrm{~m}$ or less in the vicinity of cloud top, and the total vertical domain extends to a distance well above the cloud layer (typically to $1500 \mathrm{~m}$ or higher). Modeling groups were also asked to standardize their thermodynamic calculations so that the initial state corresponded to a cloud layer between 600 and $800 \mathrm{~m}$ with $q_{l} \approx 0.45 \mathrm{~g} \mathrm{~kg}^{-1}$ at cloud top. Statistics summarizing the behavior of individual simulations conformed to two network common data form (NetCDF) templates: one for time series statistics documenting the state of the simulation at frequent intervals, the other providing profile statistics valid at the initial time and averaged over the eight 30 -min intervals that span the simulation period. Output templates are provided in an appendix, as are thumbnail plots of all the time series data and the profile statistics for the last hour. The numerical values of the statistics from individual simulations, as well as a summary of these statistics, are available in NetCDF format online through the auspices of the Data Integration for Model Evaluation (DIME) project (http://gcss-dime.giss.nasa.gov).

\section{c. Analysis methods}

Overall, scores of simulations were performed at the standard and finer resolutions and over a larger domain. The University of California, Los Angeles (UCLA) group alone performed dozens of simulations at the prescribed resolution, in addition to one (described by ZHU) that ran for $48 \mathrm{~h}$, another that was on a domain with a fourfold increase in area, and a third for which the horizontal grid spacing was refined by a factor of 2. These simulations bore out the representativeness of the results at the chosen horizontal resolution (35 m) and grid size (96 points), as well as the relatively short simulation period of only $4 \mathrm{~h}$.

Results are typically presented in terms of modified Tukey box plots; that is, the full range of the data is shown by light shading, and that half of the distribution centered on the median value is shown by dark shading (i.e., for a distribution divided into quartiles the dark shading is delineated by the first and third quartile). The solid line represents the ensemble mean. Because trying to combine all the simulations performed by each group into an ensemble would unfairly weight the ensemble toward those few groups who ran a great number of simulations, each group was asked to provide at most two simulations to be considered as part of what we call the master ensemble, with the caveat that the two simulations had to differ from one another in some formulaic way, for instance by representing an important process using a changed numerical method, or by changing how unresolved motions are parameterized. This selection resulted in a master ensemble of 16 simulations, which forms the basis of most of our analysis, with additional simulations being drawn upon as necessary.

Most of the analysis is performed on the basis of averages over the last $60-120 \mathrm{~min}$ of the simulation, during which time the simulation statistics were relatively stationary. Eddy turnover times (as measured by the ratio between the PBL height and the convective velocity scale, $w_{*}$ ) are of order of $10 \mathrm{~min}$, which implies that for each simulation somewhere between 6 and 12 independent realizations are sampled. Past studies have shown that for averaging times significantly longer than the eddy turnover time, differences among independent, identically configured simulations are negligible (e.g., Stevens et al. 2001).

\section{Results}

The temporal evolution of liquid water path, cloud fraction, and vertically integrated turbulence kinetic energy (TKE) from the master ensemble are plotted in Fig. 2. Most of the temporal evolution in the simulations is evident in the first $2 \mathrm{~h}$, with a characteristic "spinup" period evident in the first hour. During the spinup, vertically averaged TKE increases markedly as turbulent eddies develop in response to the destabilization of the PBL by surface and radiative fluxes. As the PBL turbulence increases, every simulation (to varying degrees) experiences a reduction in its liquidwater path (LWP). By the end of the first hour the LWP varies by as much as a factor of 5, with LWP $<30$ $\mathrm{g} \mathrm{m}^{-2}$ for the lowest quartile of the master ensemble. Similar reductions are also evident in the modelsimulated cloud fractions with the lowest quartile dropping well below $100 \%$. The cloud fraction distribution also illustrates the skewness of the master ensemble, wherein the mean lies in the lowest quartile. Distributions similar to those illustrated in Fig. 2 could in principle be produced by simulations whose behavior is almost identical, but characterized by large, out-of-phase, temporal oscillations. This was not the case: The degree of stationarity in the distributions in Fig. 2 is commensurate with the stationarity of individual simulations, and the spread in the distribution reflects the spread of the time-averaged statistics among the individual simulations. The stationarity between the second and fourth hour substantiates our earlier claim and is why we concentrate our analysis on the latter $2 \mathrm{~h}$ of the simulations.

For the observed case, cloud fraction, as indepen- 

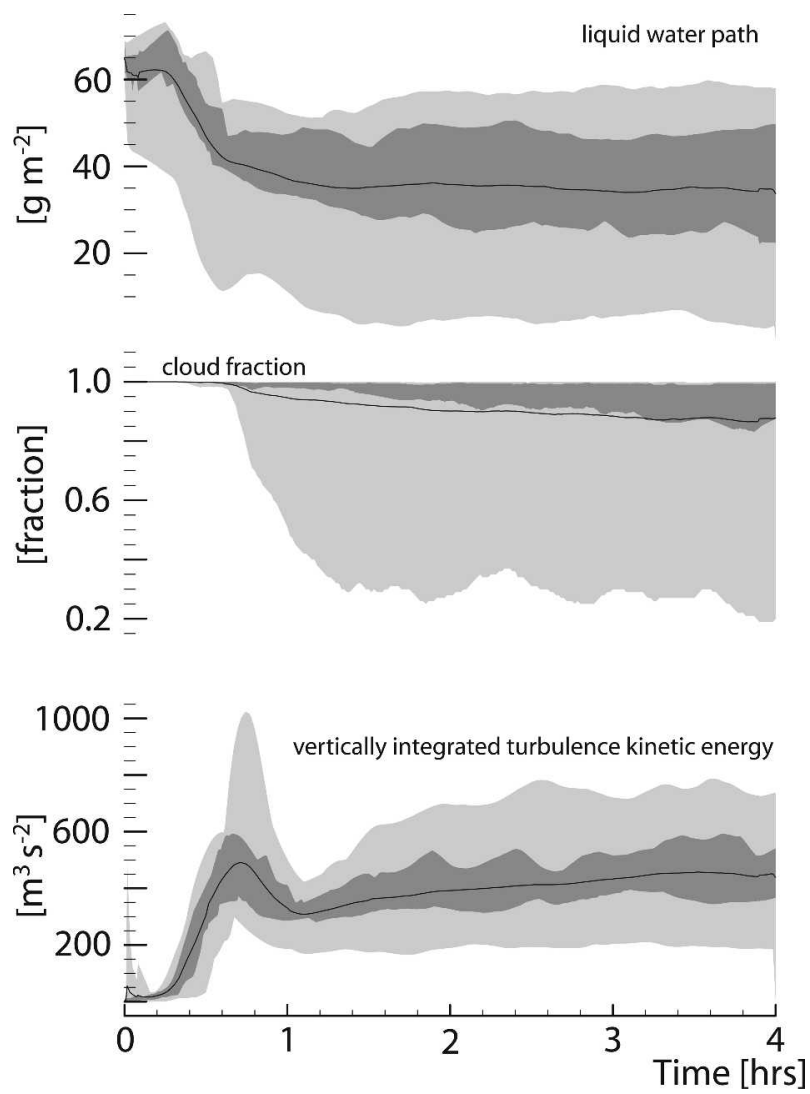

FIG. 2. Time series of master ensemble liquid water path, cloud fraction, and turbulence kinetic energy. The light shading spans the entire distribution and is delimited by the maximum and minimum value within the master ensemble at any given time. The dark shading denotes the central half of the distribution as delimited by the first and third quartiles. The line is the ensemble mean over the master ensemble.

dently measured by downward-pointing lidar and in situ data taken during cloud-top legs, was greater than $99 \%$ throughout the night. We had no direct measurements of cloud liquid water path evolution to compare to the upper panel of Fig. 2; however, estimates of the temporal evolution of cloud boundaries and liquid water profiles from the aircraft data indicate that the cloud layer maintained its thickness, and perhaps even deepened. These data, along with the distribution of the LES master ensemble, are shown in Fig. 3. In contrast, most of simulations tend to predict more broken cloud (i.e., Fig. 2) with a higher cloud base, and less liquid water than was observed. Only the upper quartile approaches the constancy in cloud fraction and tendency toward constant or increasing cloud thickness apparent in the data. ${ }^{2}$

Because most of the evolution in the cloud layer

\footnotetext{
${ }^{2}$ Note that his "upper" quartile all has unity cloud fractions, which makes it difficult to discern in the middle panel of Fig. 2.
}

is evident relatively early in the simulation, one is tempted to argue that the divergence among the simulations is the result of the spinup process and need not reflect the inherent dynamics of any particular simulation. From (3) and Fig. 1 the radiative forcing is sensitive to the cloud amount, so that simulations that tend to diminish the cloud layer during the spinup period will tend to have less radiative driving. On the one hand, this could be viewed as a negative feedback in that less radiative driving should lead to less entrainment and hence less entrainment drying, which tends to evaporate the cloud. On the other hand, relatively less radiative driving favors decoupling, which we associate here with a differentiation between the properties of the cloud and subcloud layer and which, roughly speaking, is thought to occur when the radiative forcing becomes small compared to forcings due to surface moisture fluxes (Bretherton and Wyant 1997; Stevens 2000a; Lewellen and Lewellen 2002). To examine this possibility we initialized a version of the UCLA model (UCLA-1, details of which are discussed further below), which tended to produce a relatively thinner more-broken cloud, from a fully developed flow produced by a version of that same model (UCLA-0) that better maintained the cloud. Two tests were performed, one with the UCLA-1 model branching from the UCLA-0 solutions after $1 \mathrm{~h}$, the second with the branch point after $2 \mathrm{~h}$. In both cases the UCLA-1 solutions immediately tended toward solutions with a thinner, more broken cloud. Based on this we conclude that the differences among the simulations were not an artifact of the spinup procedure, but reflected truly formulaic differences among the members of the master ensemble. This conclusion was bolstered by a number of additional tests performed with other models, wherein various modifications were made to generate a more gradual spinup. These too indicated that the divergent behavior among models (i.e., whether it tended to maintain or dissipate the cloud) was not significantly aggravated by the spinup procedure.

Further insight into the behavior of the simulations is provided by the vertical profiles of $\theta_{l}$ and $q_{t}$, which along with the liquid water mixing ratio $q_{l}$, are overlaid with the aircraft data in Fig. 4. In addition to maintaining significantly less liquid water, many simulations tend to evolve more rapidly to a warmer state, with evidence of decoupling in the mean profiles. This is particularly evident in the envelope of the $q_{t}$ profile distribution, which suggests that the reduction in the cloud amount is due to a drying of the cloud layer, as might be expected for decoupled boundary layers. Although this point is made more definitively later, here we simply state that those simulations that maintain the 


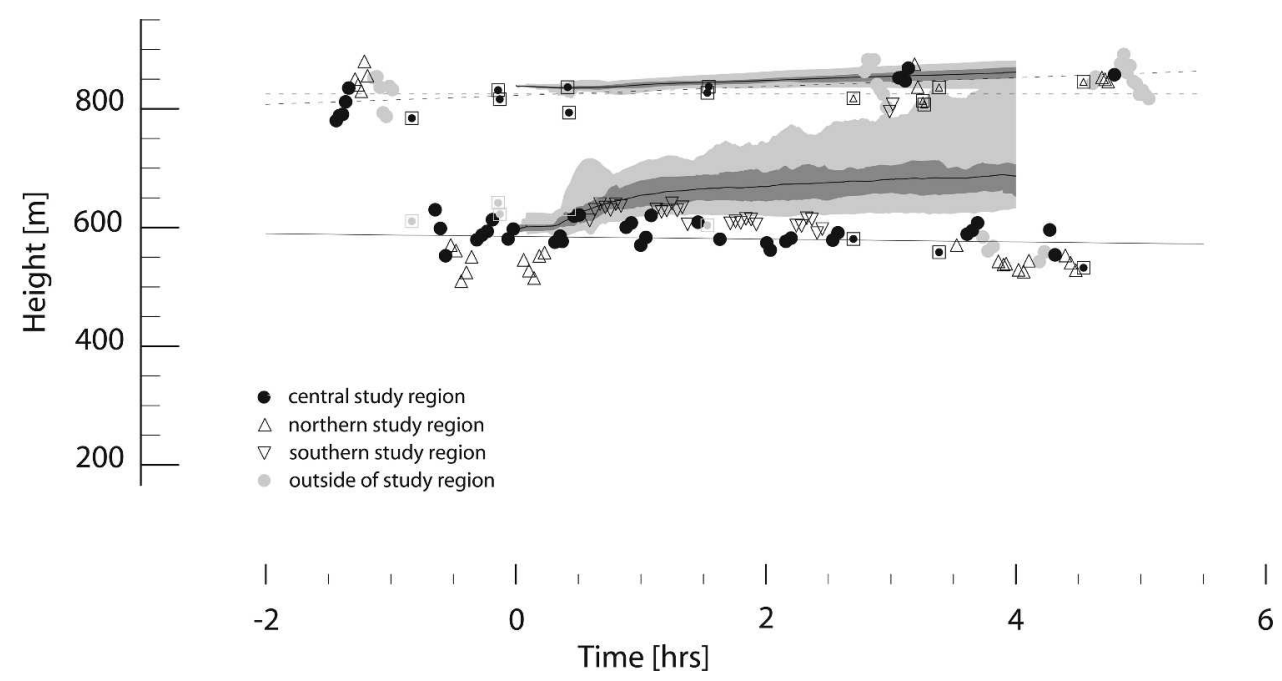

FIG. 3. Time series of cloud boundaries of master ensemble, with shading as in Fig. 2. Markers identify cloud boundaries following the analysis of Stevens et al. (2003b), wherein the data were tagged for their Lagrangian coherency. The LES case here was designed to match the observations over the central study region where most of the data were collected. In this figure unboxed markers denote cloud-base estimates from the lifting condensation level calculated using in situ data collected below cloud top or cloud-top estimates derived from lidar and radar backscatter. Boxed markers indicate estimates of cloud base and top from aircraft soundings. The straight solid line denotes the best-fit line to the cloud base over the central study region; it descends at a rate of $2 \mathrm{~m} \mathrm{~h}^{-1}$. Dashed lines are fits by eye to cloud-top data.

deepest cloud, and which are in relatively good agreement with the data in the rightmost panel of Fig. 4, also tend to be the coolest, and most well mixed, while those with the driest cloud layer also tend to have the moistest subcloud layer. The minimum in $q_{t}$ evident in some of the simulations just above cloud top is a numerical artifact that arises from phase errors produced by some advection schemes in the presence of sharp gradients. It is usually accompanied by a spurious maximum in the buoyancy flux just above cloud top.

The ensemble representation of the PBL turbulent structure, as measured by the vertical velocity variance, $\sigma_{w}^{2} \equiv \overline{w^{\prime} w^{\prime}}$, and the third moment, $\overline{w^{\prime} w^{\prime} w^{\prime}}$, is shown in the center and right panels of Fig. 5. The main source of
TKE, the buoyancy flux, is plotted in the left panel. Decoupling is also often associated with negative buoyancy fluxes near cloud base, and a cloud-base minimum in $\overline{w^{\prime} w^{\prime}}$. By these more dynamic measures about half of the simulations show evidence of decoupling.

In marked contrast the in situ and radar data suggest that the PBL remains well mixed with a single welldefined peak in $\overline{w^{\prime} w^{\prime}}$ near cloud base. This peak is nearly twice as large as the ensemble average at that level. Indeed, only the most energetic members of the LES ensemble come close to approaching the observed energetics of the layer. As exemplified by the UCLA-0 profile (which is discussed in more specific detail subsequently), these simulations also tend to maintain a
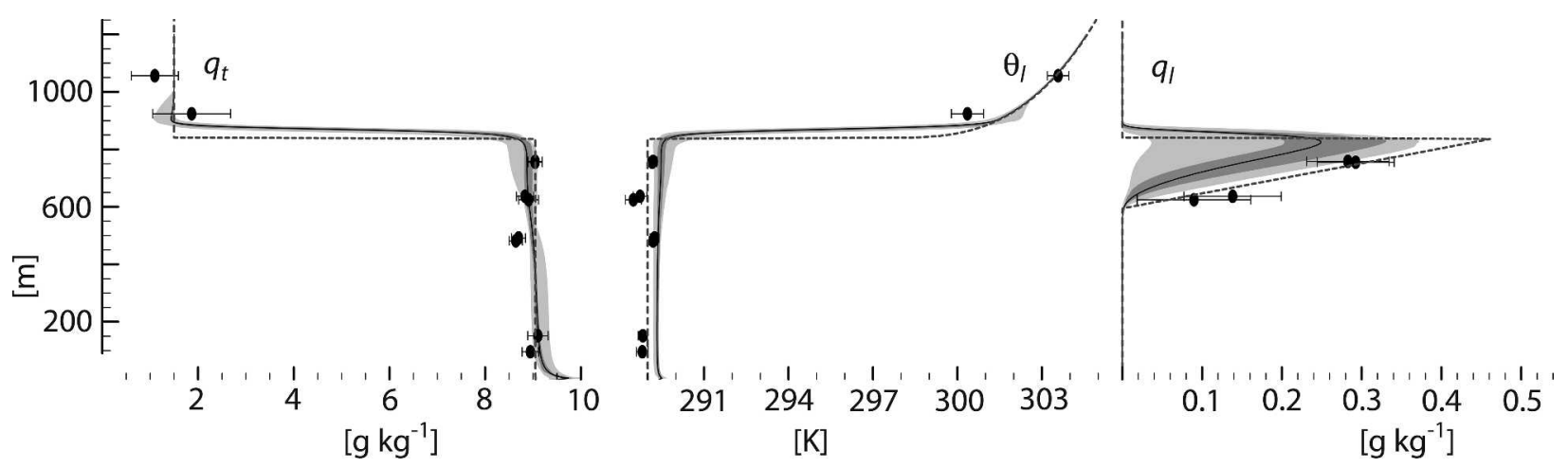

FIG. 4. Profile of mean state of specific humidity and temperature at initial time (dashed lines), as observed (points), and from master ensemble averaged over the fourth hour (solid lines). The shading is as in Fig. 2 and as described in the text. 


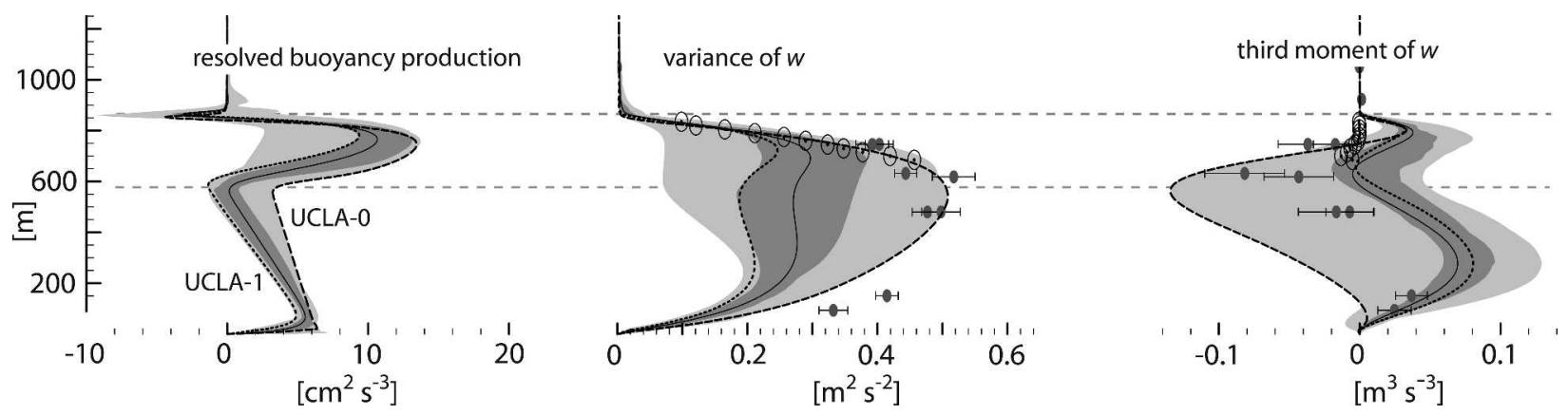

FIG. 5. Profile of vertical velocity statistics—(left) resolved buoyancy production, (middle) variance of $w$, and (right) third moment of $w$-from master ensemble averaged over the fourth hour. Markers indicate estimates of vertical velocity second and third moments as derived from in situ (solid with bar) and radar (circle-dot). Details of data analysis provided by Stevens et al. (2003a). As labeled in the left panel, the dashed lines are two simulations drawn from the master ensemble: UCLA-0 (long dash) and UCLA-1 (short dash). Horizontal dashed lines delimit cloud area. The shading is as in Fig. 2 and as described in the text.

relatively large and positive flux of buoyancy near cloud base. Considerable variability is also evident in the third moment of $w$, which measures the structure of the turbulence. For instance, negative skewness is indicative of strong downdrafts as might be expected in a flow driven predominantly by radiative cooling, where positive skewness is characteristic of surface-based and cumulus convection. The simulations in the master ensemble differ in the sign of this moment. As decoupling becomes more evident there is the expectation that the turbulence will become more surface driven and have more positive third moments through the bulk of the PBL, and thus the disparity in $\overline{w^{\prime} w^{\prime} w^{\prime}}$ among the simulations may further measure the degree of decoupling. Clearly some of the simulations show evidence of capturing the observed negative values in $\overline{w^{\prime} w^{\prime} w^{\prime}}$ near cloud base, although all show more positively skewed circulation than observed in the upper $100 \mathrm{~m}$ of the cloud layer. Previous simulations of this case whose forcing was based on (3) with $F_{0}=50 \mathrm{~W} \mathrm{~m}^{-2}$ and $F_{1}=$ $D=0$, failed to represent the region of negative skewness near cloud base (Stevens et al. 2003b). Sensitivity studies show that a better representation of this feature accompanies the more realistic representation of the forcing embodied by including all of the terms in (3).

There is a strong relationship between model-tomodel variations in turbulence, decoupling, and subcloud buoyancy fluxes. Simulations that produce the thinnest clouds, as for instance measured by LWP, also tend to produce less turbulence in the cloud layer and embody larger gradients in moisture within the PBL. This is shown by Fig. 6, where we plot the moisture change across the PBL, as measured by

$$
\delta q_{t} \equiv 0.01\left(\int_{100 m}^{200 m} q_{t} d z-\int_{700 m}^{800 m} q_{t} d z\right)
$$

the minimum value of the resolved buoyancy flux, and the maximum value of the vertical velocity variance $\sigma_{w}^{2}$ versus the LWP averaged over the last hour for each of the simulations. The master ensemble includes two simulations with markedly less LWP, smaller maximum values of $\sigma_{w}^{2}$, and the development of large gradients in $q_{t}$. The remaining distributions are more uniformly distributed from one extreme where the PBL is clearly decoupled, with a pronounced negative buoyancy flux at the top of the subcloud layer, substantial gradients in $q_{t}$, and modest values of $\sigma_{w}^{2}$ to the other where there is little or no evidence of decoupling and $\sigma_{w}^{2}$ is larger. The observations are closer to this latter extreme. It is also worth noting that the differentiation of the vertical structure of the layer, as for instance measured by $\delta q_{t}$, leads to a marked reduction in cloudiness: whereas the simulated LWPs differ by more than a factor of 5 , differences in the LWP of perfect mixed layers constructed using the mean thermodynamic properties of the simulations would only differ by a factor of 2 .

Because the radiative driving of the simulations depends on both the spatial distribution of cloud water and its amount [i.e., from (3) it saturates for large local liquid water paths] it is worthwhile to establish the correlation between the radiative driving and the liquid water path. We estimate the former as $\left[F_{\text {rad }}\right]$ where the square brackets denote a difference across the bulk of the PBL, that is,

$$
(\chi) \equiv \chi\left(z_{i_{+}}\right)-\chi(0)
$$

where $\chi$ is some arbitrary field and $z_{i+} \equiv z_{i}+\epsilon$. For this calculations we take $\epsilon=5 \mathrm{~m}$, which is equal to the grid spacing at cloud top in most models. The results are given by the filled symbols in the top panel of Fig. 7 . They suggest that the tendency for the more decoupled simulations to be less turbulent reflects the fact that they are driven less vigorously. 

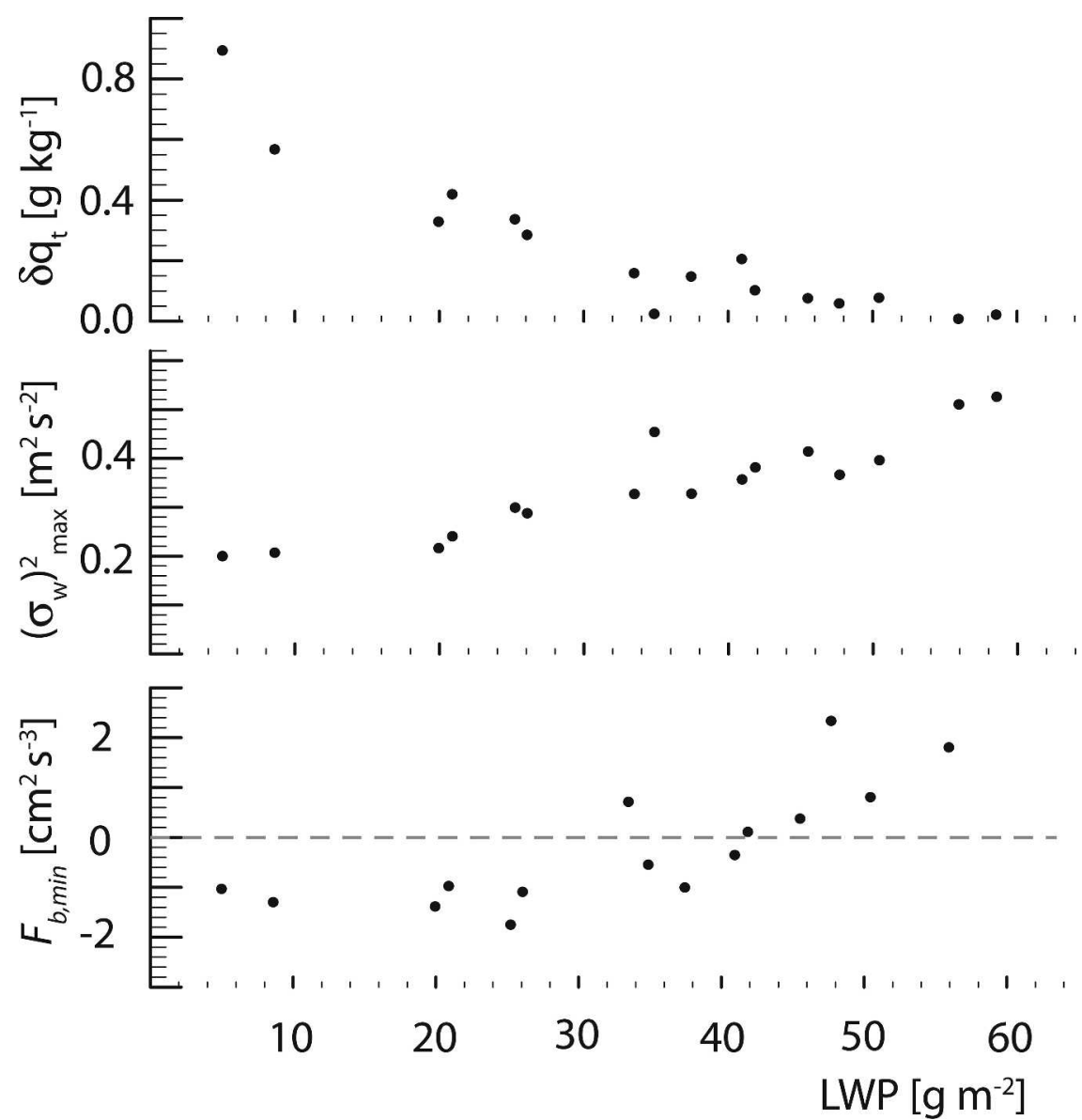

FIG. 6. Profile metrics plotted vs LWP for all members of the master ensemble: (top) Surface minus cloud layer specific humidity $\delta q_{i}$; (middle) vertical velocity variance, $\left.\sigma^{2}\right|_{\max }$; (bottom) minimum buoyancy flux $F_{b, \min }$.

Despite the clear dependence of the radiative forcing on the LWP, the solid circles in the middle panel of Fig. 7 show that among the simulations there is relatively little sensitivity of the entrainment velocity $E$ to LWP, and hence $\left[F_{\text {rad }}\right]$. Because we think of stratocumulus as being radiatively driven, with the entrainment rate depending primarily on $\left[F_{\mathrm{rad}}\right]$ this result is surprising. Its interpretation might provide some insight into why the simulations show such varied propensities to decouple. If we denote the flux of some quantity $\chi$ by $F_{\chi}$,

$$
F_{\chi} \equiv \overline{w^{\prime} \chi^{\prime}},
$$

we can associate decoupling with the tendency of the buoyancy flux $F_{b}$ to become negative below cloud base (Turton and Nicholls 1987; Bretherton and Wyant 1997). Because $F_{b}$ is positive at the surface, $d F_{b} / d z$ must be negative in the subcloud layer, which, because $F_{b}$ can be expressed as a linear combination of $F_{q_{t}}$ and $F_{\theta_{l}}$, constrains the fluxes of the thermodynamics state variables, $\theta_{l}$ and $q_{t}$. In the present case, where surface moisture and heat (and hence buoyancy) fluxes are positive and the former, $F_{q}$, changes little with height, the latter, $F_{\theta}$, hence must decrease with height-the PBL must warm. For fixed surface heat fluxes, the rate of warming, and hence the propensity to decouple, is determined by the relative contributions of entrainment warming versus radiative cooling. Figure 7 suggests that the more decoupled simulations maintain the same entrainment warming despite a decrease in the radiative cooling of the layer. This would imply that among the simulations the slope of $F_{\theta_{l}}$ in the subcloud layer should vary significantly while the slope of $F_{q_{t}}$ should be more approximately constant, as is indeed the case (e.g., Fig. $\mathrm{C} 1$ in appendix $\mathrm{C}$ ).

To make these arguments more precise we can compare the entrainment warming to the radiative cooling by evaluating the ratio

$$
\alpha=\frac{E \Delta \theta_{l}}{\left[F_{\mathrm{rad}}\right]},
$$

where for any variable $\chi$

$$
\Delta \chi \equiv \chi\left(z_{i_{+}}\right)-\langle\chi\rangle, \text { with }\langle\chi\rangle \equiv \frac{1}{z_{i_{+}}} \int_{0}^{z_{i_{+}}} \chi .
$$



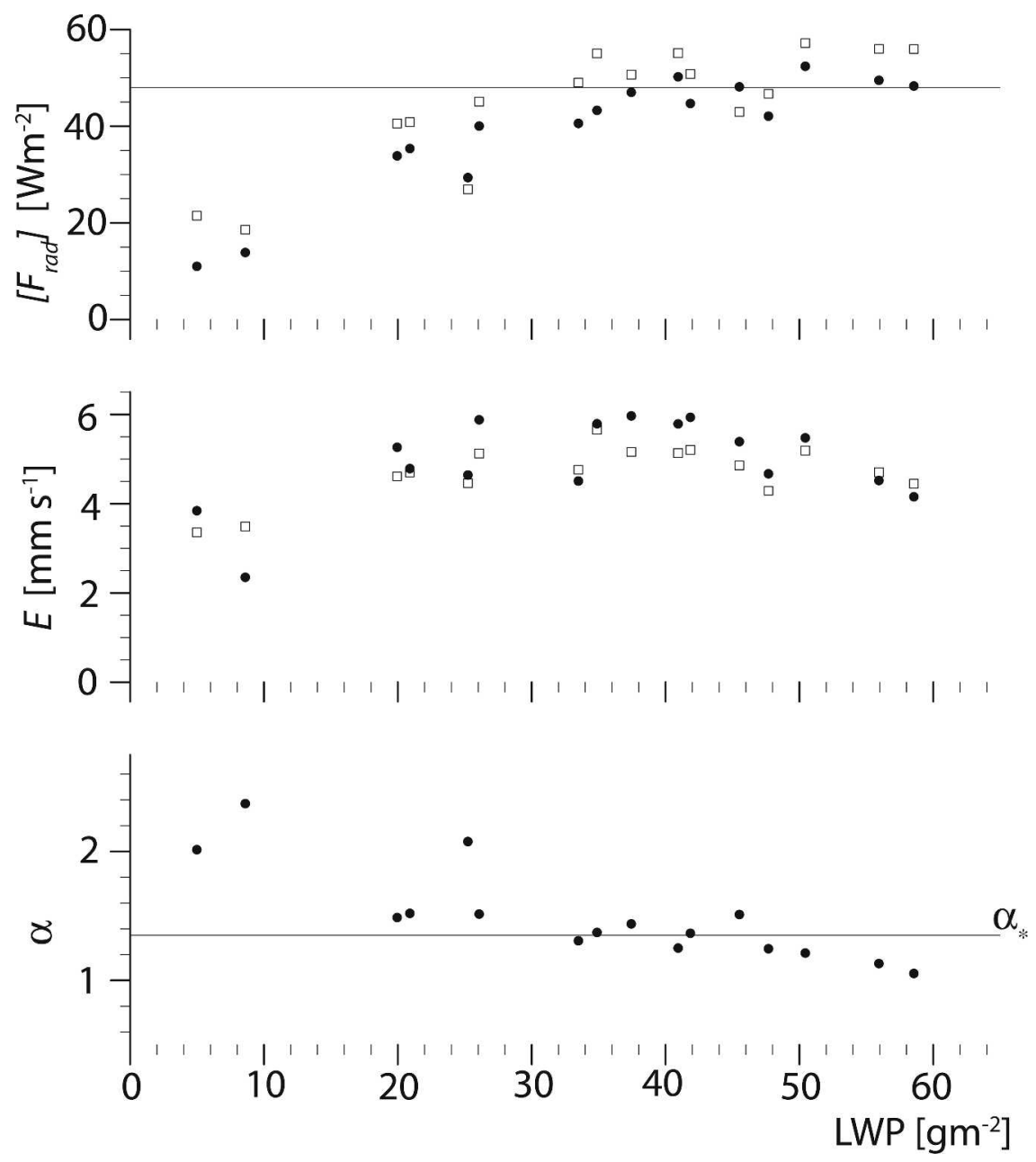

FIG. 7. Various flow statistics plotted vs LWP for the master ensemble. (top) Radiative flux divergence across the entire PBL estimated directly (solid circles) or from heat and moisture budgets (open circles); solid gray line denotes maximum flux divergence $F_{0}-F_{1}=48 \mathrm{~W} \mathrm{~m}^{-2}$ due to the presence of cloud. (middle) Entrainment rate, $E$, as estimated by the time rate of change of $z_{i}$ (solid circles) or by the moisture budget (open circles). (bottom) Nondimensional entrainment rate $\alpha$. The solid gray line on the last panel indicates, $\alpha_{*}$, the mixed-layer estimate of a decoupling threshold.

The quantity $\alpha$ can also be interpreted as a nondimensional entrainment rate, or radiative entrainment efficiency. For $\alpha \gg 1$ radiative processes contribute negligibly to the heat budget, $\alpha=1$ indicates a balance between entrainment warming and radiative cooling, while the relative role of entrainment warming diminishes with decreasing $\alpha$.

An evaluation of $\alpha$ directly from (8) is sensitive to how one defines $E$ and the value of $\epsilon$. Such sensitivities are due in large part to large radiative heating rates in the layer just above cloud top, as modeled by the third term in our parameterization of radiative fluxes [e.g., Eq. (3)], which leads, in turn, to commensurate changes in $\theta_{l}$. Sharp and coupled changes in $\theta_{l}$ and $F_{\text {rad }}$ mean that attempts to evaluate (8) directly are sensitive to the structure of the inversion as represented by different models, and hence $\epsilon$. The bulk budgets of heat and moisture,

$$
\begin{aligned}
& z_{i} \frac{d\left\langle\theta_{l}\right\rangle}{d t}=-\left(F_{\theta_{l}}\right)-\left[F_{\mathrm{rad}}\right]=E \Delta \theta_{l}+F_{\theta_{l}}(0)-\left[F_{\mathrm{rad}}\right] \\
& z_{i} \frac{d\left\langle q_{t}\right\rangle}{d t}=-\left(F_{q_{t}}\right)=E \Delta q_{t}+F_{q_{t}}(0)
\end{aligned}
$$

where $E=d z_{i_{+}} / d t$ provide a more robust alternative. Solving for $E$ from (10) and for $\left[F_{\text {rad }}\right]$ from (11) yields an expression for $\alpha$ largely in terms of bulk quantities: 


$$
\alpha=\frac{\left[z_{i} \frac{d\left\langle q_{t}\right\rangle}{d t}-F_{q_{t}}(0)\right] \Delta \theta_{l}}{\left[z_{i} \frac{d\left\langle q_{t}\right\rangle}{d t}-F_{q_{t}}(0)\right] \Delta \theta_{l}-\left[z_{i} \frac{d\left\langle\theta_{l}\right\rangle}{d t}-F_{\theta_{l}}(0)\right] \Delta q_{t}} .
$$

The lower panel of Fig. 7 shows how $\alpha$ estimated in this fashion (with $\epsilon=50 \mathrm{~m}$ ) covaries with LWP across the master ensemble. The values of $E$ and [ $\left.F_{\text {rad }}\right]$ estimated from the bulk budgets are also plotted as open symbols in the upper panels. They compare favorably with direct estimates, although the need to define a thick interface, that is, $\epsilon=50 \mathrm{~m}$ so as to span the interfacial layer (cf. Moeng et al. 2005), necessarily introduces a significant component of the clear air cooling into the expression for $\left[F_{\text {rad }}\right]$. This is evident in the offset between the two estimates of $\left[F_{\text {rad }}\right]$; fortunately it does not alter the trend among the models. Figure 7 thus quantifies the diminishing role of radiative cooling as the simulations become increasingly decoupled.
If we use the criterion that a mixed-layer estimate of $F_{b}$ vanishes at cloud base as a measure of decoupling, then this decoupling threshold can be expressed as a critical value of $\alpha$, which we denote by $\alpha_{*}$. To estimate $\alpha_{*}$ we note that for a quasi-steady subcloud layer, with negligible radiative flux divergence,

$$
F_{b}(z)=c_{1}\left[F_{\theta_{l}}(0)+z \frac{d F_{\theta_{l}}}{d z}\right]+c_{2}\left[F_{q_{t}}(0)+z \frac{d F_{q_{t}}}{d z}\right],
$$

where $c_{1}$ and $c_{2}$ are thermodynamic constants, which for unsaturated fluctuations are equal to $0.034 \mathrm{~m} \mathrm{~s}^{-2}$ $\mathrm{K}^{-1}$ and $5.9 \mathrm{~m} \mathrm{~s}^{-2}$, respectively. From (13), $F_{b}$ vanishes at cloud base (denoted by $z=z_{b}$ ) if

$$
\frac{d F_{\theta_{l}}}{d z}=-\frac{1}{z_{b}}\left\{F_{\theta_{l}}(0)+\frac{c_{2}}{c_{1}}\left[F_{q_{t}}(0)+z_{b} \frac{d F_{q_{t}}}{d z}\right]\right\} .
$$

Because in the subcloud layer $d\left\langle\theta_{l}\right\rangle / d t=-d F_{\theta_{l}} / d z$ it follows from (12) and (14) that

$$
\alpha_{*}=\frac{\left[F_{q_{t}}(0)-z_{i} \frac{d\left\langle q_{t}\right\rangle}{d t}\right] \Delta \theta_{l}}{F_{q_{t}}(0) \Delta \theta_{l}\left(1+\frac{z_{i}}{z_{b}} \frac{c_{2}}{c_{1}} \frac{\Delta q_{t}}{\Delta \theta_{l}}\right)-z_{i} \frac{d\left\langle q_{t}\right\rangle}{d t} \Delta \theta_{l}\left(1+\frac{c_{2}}{c_{1}} \frac{\Delta q_{t}}{\Delta \theta_{l}}\right)-F_{\theta_{l}}(0) \Delta q_{t}\left(1-\frac{z_{i}}{z_{b}}\right)}
$$

is a threshold for decoupling. For the conditions of RF01, in which most simulations yielded $d\left\langle q_{t}\right\rangle / d t \ll F_{q_{t}}$ ' $z_{i}, \alpha_{*}$ can be written entirely in terms of specified quantities, that is, surface fluxes. For this special case we find that $\alpha=\alpha_{*}=1.35$ should approximately separate decoupled from well-coupled solutions. Overall the scatter of $\alpha$ across the simulation ensemble (shown in the bottom panel of Fig. 7, with $\alpha_{*}$ being indicated by the dashed line) appears to support these arguments. Moreover, using the data from Fig. 4 of Stevens et al. (2003a) the observations from RF01 suggest that $\alpha \approx 1$, which is consistent with both the well-mixedness of the observed layer, and the tendency of those simulations with the lower values of $\alpha$, to best represent it.

If $E$ scales with [ $F_{\text {rad }}$ ] then one would expect $\alpha$ to be constant. This is arguably the case for the best-mixed simulations, that is, those with LWP $>50 \mathrm{~g} \mathrm{~m}^{-2}$; it may also be the case for a single configuration of any particular code, run for cases with varied radiative forcings. In contrast, the remarkable correlation between $\alpha$ and LWP (especially evident for LWP $<50 \mathrm{~g} \mathrm{~m}^{-2}$ ) in Fig. 7 quantifies the tendencies for the more decoupled simulations to result from their ability to maintain their rate of entrainment despite the diminishment of the radiative forcing. Why and how they do this is key to understanding why their solutions diverge so markedly from the observations. Clues are provided in Table 1, where additional flow statistics, and configuration details, of individual simulations are provided. Focusing on the UCLA- 0 and UCLA- 1 simulations we note that the UCLA-1 simulation (its base, or standard, configuration) has a significant subgrid component to the total $\theta_{l}$ flux at the inversion. The implication is that much of the entrainment flux by that configuration of the code is being carried by the subgrid model, which is the most poorly justified model component, the implication being that the amount of mixing it generates is less likely to be constrained by the intensity of the PBL-scale circulations. This motivated experiments where different approaches were employed to regulate the behavior of the subgrid-scale (SGS) model. These ranged from using different stability or length scale formulations to limit mixing at the inversion to simply using the monotone property of the scalar advection scheme to carry the dissipation of scalar variance and turning off the subgrid model for scalars entirely. The latter, and most drastic change is manifest in the UCLA-0 model configuration. This configuration markedly changes the energetic profiles (Fig. 5) as compared to the UCLA-1, or standard configuration. The impact of these changes is 
TABLE 1. Members of master ensemble and various diagnostics/ configuration specifications: LWP (vertically integrated liquid water path) over last hour; $\left.F_{\theta_{l}}\right|_{\text {sgs }}$ denotes the SGS contribution to the minimum $\theta_{l}$ flux (in energetic units) near the inversion; SGS models are either Deardorff (1980b), Smago (for SmagorinskyLilly), Smago-MM indicating Smagorinsky with the MacVean and Mason (1990) stability correction, or in the case of DHARMA-0 a dynamic model. The * denotes the SGS model was modified so that the dry measure of stability was used at cloud top. Advection schemes for scalars were either variance diminishing (denoted $\mathrm{M}$, for monotone) or variance preserving ( $\mathrm{C}$ for centered or positive definite, PS for pseudospectral).

\begin{tabular}{lcrll}
\hline \multicolumn{1}{c}{ Model } & $\begin{array}{c}\text { LWP } \\
\left(\mathrm{g} \mathrm{m}^{-2}\right)\end{array}$ & $\begin{array}{c}F_{\theta_{l}} \mathrm{l}_{\mathrm{sgs}} \\
\left(\mathrm{W} \mathrm{m}^{-2}\right)\end{array}$ & \multicolumn{1}{c}{ SGS model } & $\begin{array}{c}\text { Scalar } \\
\text { advection }\end{array}$ \\
\hline UCLA-0 & 59 & 0.0 & None for scalar & $\mathrm{M}$ \\
DHARMA-0 & 56 & -0.8 & Dynamic & $\mathrm{M}$ \\
COAMPS-0 & 50 & -2.1 & Deardorff & $\mathrm{C}$ \\
NCAR-1 & 46 & -1.3 & Deardorff & $\begin{array}{c}\text { PS-Horiz, } \\
\end{array}$ \\
MPI-1 & 47 & -1.0 & Deardorff* & $\mathrm{C}$ \\
WVU-0 & 42 & -10.4 & Deardorff* & $\mathrm{M}$ \\
DHARMA-1 & 41 & -17.9 & Smag & $\mathrm{C}$ \\
RAMS & 37 & 0.2 & Deardorff & $\mathrm{C}$ \\
SAM & 35 & -10.4 & Deardorff & $\mathrm{M}$ \\
COAMPS-1 & 33 & -14.1 & Smag-MM & $\mathrm{M}$ \\
UCLA-1 & 26 & -21.5 & Smag & $\mathrm{M}$ \\
NCAR-0 & 25 & -3.7 & Deardorff & $\mathrm{M}$ \\
WVU-1 & 21 & -16.7 & Deardorff & $\mathrm{M}$ \\
METO & 21 & -24.6 & Smag-MM & M \\
MPI-0 & 9 & -7.6 & Deardorff & $\mathrm{M}$ \\
IMAU & 5 & -22.1 & Deardorff & M \\
\hline
\end{tabular}

further illustrated by cross sections from the last time step of higher-resolutions versions of the UCLA-0 and UCLA-1 simulations in Fig. 8. UCLA-0 produces a very well mixed simulation with a thicker, more horizontally homogeneous cloud layer, in better agreement with the data.

Similar sensitivities are also evident among the other models. For instance, centered schemes tend to better represent the inversion (albeit with some unphysical artifacts such as overshooting), both directly because they are nondiffusive, but also indirectly because a stronger inversion also limits SGS mixing. Changes, or features, of the SGS model that helped limit the mixing at the cloud-top interface also have a clear impact on other simulations. This is evident for the Distributed Hydrodynamic Aerosol and Radiative Modeling Application (DHARMA) model, wherein their implementation of a dynamic SGS model naturally (i.e., with out any ad hoc modifications; cf. UCLA-0) shuts down the SGS fluxes at the inversion, resulting in a better simulation. To a lesser extent, similar behavior is evident in the West Virginia University (WVU) simulations. The additional length scale correction introduced into the Deardorff (1980b) SGS model further limits cloud-top mixing and may explain why the COAMPS- 1 simulation is more dissipative than COAMPS-0. That said, these inferences are just that, inferences. Because the numerical methods employed are quite varied and because the behavior of the SGS model is so deeply intertwined with the representation of the small-scale flow it is difficult to separate numerical from SGS effects (e.g., Ghosal 1996) and thus associate particular results with simple statements about the character of the host model.

\section{Discussion}

Have we made progress? After nearly a decade of effort the spread among LES is, for the case of some important parameters (e.g., LWP), as large as it was in the prior study by Moeng et al. (1996). Moreover, the hypothesis that the entrainment rate is controlled by the transport properties of large eddies in a manner insensitive to the detailed fluid-dynamical mechanism of entrainment is challenged by our results. At least for this case the basic character of the simulation is remarkably sensitive to details of the simulations. Nonetheless, in contrast to the situation 10 years ago, we now have a good idea of the right answer, and are able to say that many important features of the observed cloud layer can be represented by the LES, if efforts are made to minimize mixing at the cloud-top interface. Caution however is warranted: Simulations in better accord with the data do not necessarily indicate that entrainment is better represented by a particular model, or model configuration. Rather, if for a given forcing a simulation can be made to predict the correct entrainment rate, other aspects of the simulation are likely to be in better accord with the data. This point gains emphasis from a series of simulations using the UCLA- 0 model configuration, for which no subgrid model was employed for the scalars, but with varying vertical grid spacing near cloud top. In addition to the base cases whose vertical grid spacing is $5 \mathrm{~m}$ through a $100-\mathrm{m}$ zone spanning cloud top, three additional simulations were performed with $\Delta z=10,2$, and $1 \mathrm{~m}$, respectively. The results from these simulations are shown in Fig. 9. With increasing vertical resolution across cloud top, numerical diffusion is reduced. For instance, first-order advection schemes (which monotone schemes approach in the limit of sharp interfaces) have an effective diffusivity that can be expected to scale as $w_{\mathrm{rms}} \Delta z$, where $w_{\mathrm{rms}}$ is the rms velocity of the local interface. By reducing the effective diffusion at cloud top, better-mixed and more energetic solutions (see also Table 2) are obtained. Here we note that the lack of a subgrid model renders the simulations sensitive to the grid; similar sensitivities are also evident in other simulations where the SGS model more naturally 


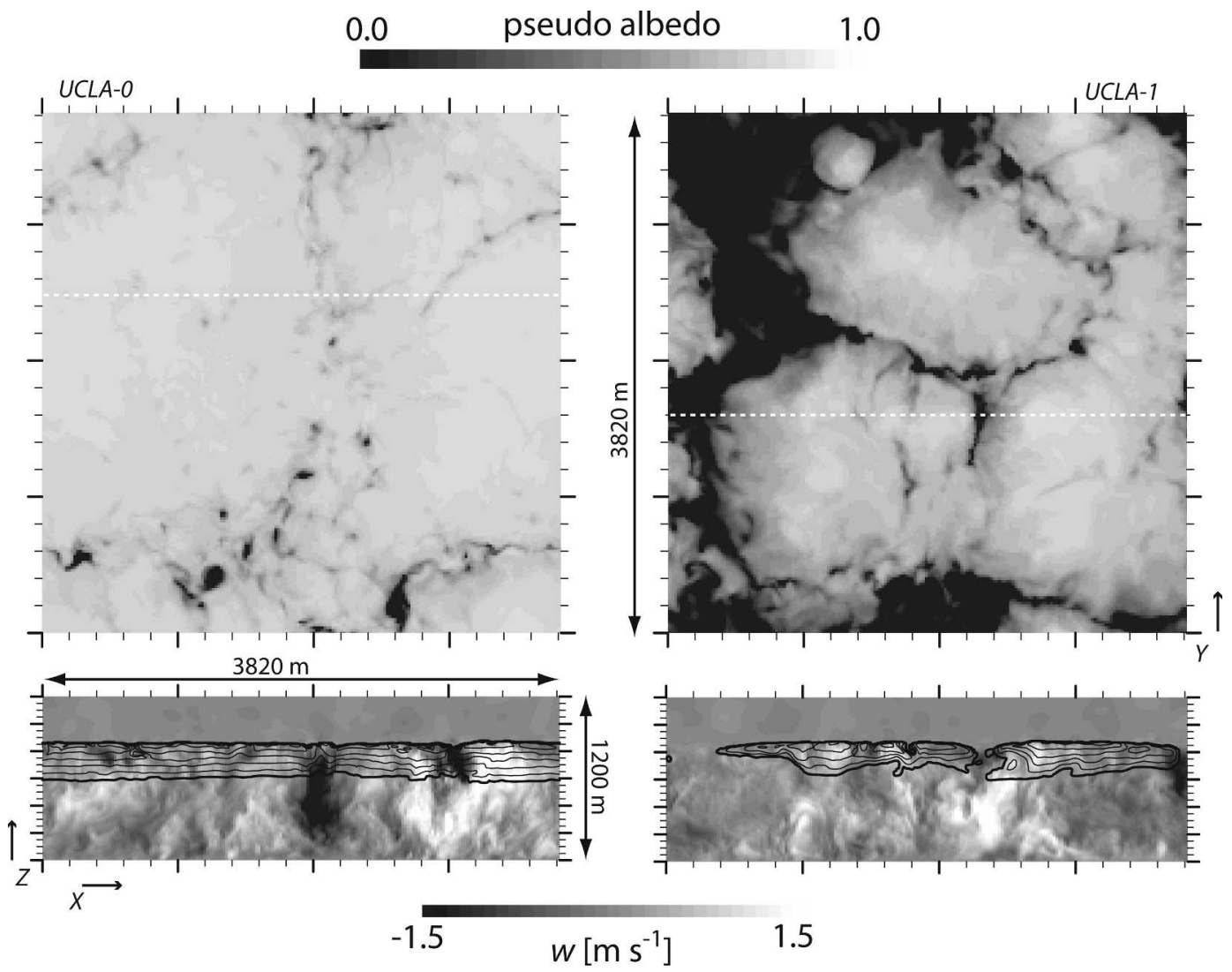

FIG. 8. (top) Visualization of flow fields from (left) UCLA-0 and (right) UCLA-1 simulations at the end of the simulation period. Shown are (top) plan-view images of the albedo estimated from the liquid water path, and (bottom) cross sections showing vertical velocity (shaded) and cloud water (contoured). The cross-section cuts are indicated by the white dashed line in plan-view plots. These fields are drawn from simulations wherein $N_{x}=N_{y}$ $=192$ and $\Delta x=\Delta y=20 \mathrm{~m}$. The change in the horizontal mesh leads to more pleasing flow visualization, but has no marked impact on the flow statistics.

shuts down at the inversion. Breaking such sensitivities is a prime motivation for including SGS physics.

It is difficult to say whether the UCLA-0 simulations with a 1-, 2-, or 5-m vertical grid near cloud top better represents the observed layer, particularly given that

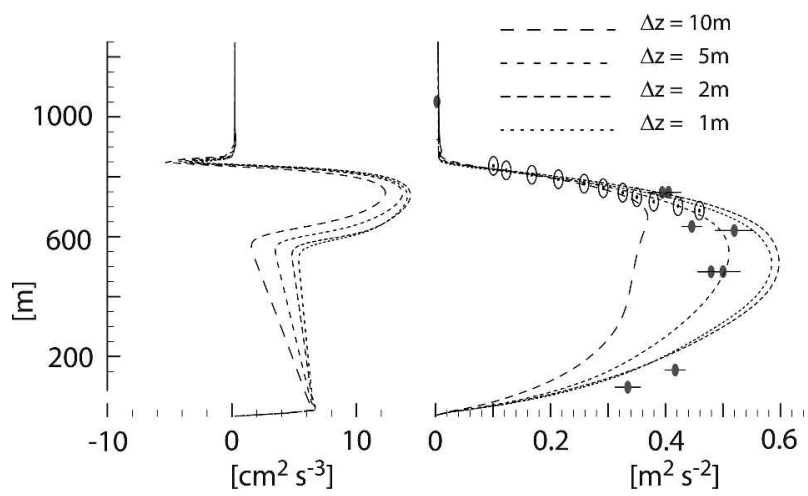

FIG. 9. Profiles of the buoyancy flux and vertical velocity (cf. Fig. 5 ) but with four different simulations by the UCLA-0 configuration of the model and different vertical grid spacings of 1,2, 5, and $10 \mathrm{~m}$. sensitivity tests with the DHARMA model suggest that the neglect of droplet sedimentation in the cloud layer leads to an overprediction of turbulence intensities, commensurate with what is shown in Fig. 9. Nonetheless it is conceivable that the simulation with the finest

TABLE 2. Scalar metrics for different configurations of UCLA-0 (no SGS on scalars) and UCLA-1 models. The last two simulations are for a different set of initial conditions as discussed in section 4.

\begin{tabular}{|c|c|c|c|c|}
\hline $\begin{array}{c}\mathrm{LWP} \\
\left(\mathrm{g} \mathrm{m}^{-2}\right)\end{array}$ & $\begin{array}{c}\delta q \\
\left(\mathrm{~g} \mathrm{~kg}^{-1}\right)\end{array}$ & $\begin{array}{c}E \\
\left(\mathrm{~mm} \mathrm{~s}^{-1}\right)\end{array}$ & $\alpha$ & Notes \\
\hline 59 & 0.02 & 4.16 & 1.04 & UCLA-0 \\
\hline 26 & 0.29 & 5.89 & 1.74 & UCLA-1 \\
\hline 42 & 0.08 & 5.03 & 1.25 & $\operatorname{UCLA}-0(\Delta z=10 \mathrm{~m})$ \\
\hline 70 & 0.00 & 4.26 & 0.91 & $\operatorname{UCLA}-0(\Delta z=2 \mathrm{~m})$ \\
\hline 74 & 0.00 & 3.75 & 0.86 & $\operatorname{UCLA}-0(\Delta z=1 \mathrm{~m})$ \\
\hline 99 & 0.01 & 4.59 & $\mathrm{n} / \mathrm{a}$ & $\begin{array}{l}\text { UCLA-0 } \\
\qquad\left(q_{t}^{+}=5.5 \mathrm{~g} \mathrm{~kg}^{-1}\right)\end{array}$ \\
\hline 82 & 0.05 & 5.82 & $\mathrm{n} / \mathrm{a}$ & $\begin{array}{l}\text { UCLA-1 } \\
\qquad\left(q_{t}^{+}=5.5 \mathrm{~g} \mathrm{~kg}^{-1}\right)\end{array}$ \\
\hline
\end{tabular}


vertical spacing near cloud top underpredicts entrainment, and hence overpredicts the strength of the large eddies because of a lack of commensurately fine horizontal resolution. In this case relatively small sensitivity to reductions in $\Delta z$ beyond $2 \mathrm{~m}$ might be less a statement of convergence and more a statement that the vertical gradients of eddies whose horizontal scale is larger than $70 \mathrm{~m}$ are then resolved, which does not guarantee that such convergence will be preserved, as finer scales are included in the horizontal. To investigate these issues one should repeat these resolution studies with an isotropic refinement. Computations with a mesh spacing of $1 \mathrm{~m}$ in all directions, on some of the largest available computers, can be anticipated in the coming years.

Resolutions studies such as these will be particularly interesting because the physics of small-scale processes in the interfacial layer are so rich. As is the case for dry convective layers driven by surface fluxes, small-scale eddies in the interfacial layer are driven by the dissipation of larger-scale plumes as they impinge upon the stably stratified interface, but quite unlike dry convective layers smaller-scale eddies can be directly driven by diabatic (radiative, evaporative) processes at cloud top. Moreover, because of the close proximity of the cloudtop radiative cooling to the inversion, this small-scale turbulence can mix some of the radiatively cooled parcels with warmer inversion air, neutralizing their negative buoyancy and thereby effectively reducing the fraction of radiative forcing that drives PBL-scale turbulence; this may explain the particular sensitivity of stratocumulus simulations to the representation of small-scale processes (Lewellen and Lewellen 1998). Because processes near cloud top should in principle be resolvable, studies with very high resolution will be central to better understanding turbulent processes in regions of great thermal stability, thereby informing the construction of more robust SGS models.

Although the above arguments suggest that simulations of stratocumulus are more sensitive to the representation of subgrid-scale physics, might RF01 be an especially sensitive case? This question arises because a recent intercomparison by Duynkerke et al. (2004), of essentially the same collection of models, showed more satisfactory agreement amongst simulations. Because the initial state for the present case satisfies the Randall (1980) and Deardorff (1980a) cloud-top entrainment instability (CTEI) criterion at cloud top, ambiguities in defining the stability of the cloud-top interface may be compounded because such a criterion is implicit in many subgrid models. The dryness of the free troposphere also implies that moisture fluxes in the cloud layer will be large and thus contribute significantly to kinetic energy production by enhancing the buoyancy

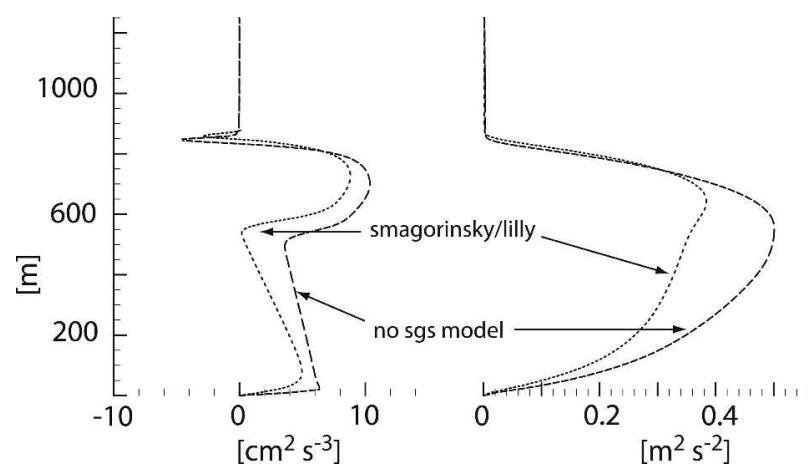

FIG. 10. Profiles of the buoyancy flux and vertical velocity (cf. Fig. 5) but for simulations with a free-tropospheric $q_{t}=5.5 \mathrm{~g} \mathrm{~kg}^{-1}$.

flux through evaporative cooling. In such a situation the layer is thought to be particularly susceptible to decoupling (Bretherton and Wyant 1997). Because the transition to a decoupled PBL appears to be relatively sharp (Stevens 2000b), the degree to which simulations fall on one or the other side of this boundary might amplify differences among simulations. To test these ideas we repeated the RF01 simulation but with freeatmospheric profiles of $q_{t}$ increased to $5.5 \mathrm{~g} \mathrm{~kg}^{-1}$ so as to render the cloud-top interface stable from the perspective of the CTEI criterion of Randall and Deardorff and to diminish the total water fluxes and hence the buoyancy flux in the cloud layer, thereby making the layer less susceptible to decoupling. Results from these simulations are shown in Fig. 10 and tabulated in Table 2. The UCLA-1 configuration of the model entrains more, produces a thinner cloud, and is less energetic. However, both simulations remain relatively well mixed and the disparity in the results is less than it was for the case with the drier free troposphere; that is, the LWP differs by only $21 \%$ for the case with a moist free troposphere, as compared to the $127 \%$ difference previously. This better agreement for this case is consistent with the robustness of the diurnal cycle intercomparison of LES presented by Duynkerke et al. (2004).

\section{Conclusions}

Data from the first research flight (RF01) of DYCOMS-II were used to reexamine the fidelity of LES in representing the turbulent structure of stratocumulus-topped boundary layers. Literally scores of simulations from 10 modeling centers were configured to represent the observed state of the PBL during RF01. Of these, 16 whose initial state and forcings conformed to the specifications outlined for the intercomparison were chosen for presentation here, with the additional simulations being used to guide our interpretation of the results. Overall we found the following: 
1) If efforts are made to reduce mixing at cloud top, either by choosing a less diffusive numerical scheme, refining the vertical grid, or limiting the effects of the subgrid model in this region, then the observed turbulent and thermodynamic structure of the layer can be reproduced with some fidelity. For such simulations, only the third moment of the vertical velocity near the top of the cloud layer showed any significant disagreement with the observations.

2) The base, or native, configuration of most simulations - that is, that which would have been used in the absence of prior knowledge of the answeroverestimated mixing at cloud top, tending toward a decoupled layer in which cloud liquid water path and turbulent intensities were grossly underestimated.

3) The sensitivity of the simulations to the representation of mixing at cloud top is amplified by particulars of this case, wherein the cloud-top interface is unstable from the perspective of the (Randall 1980; Deardorff 1980a) cloud-top entrainment instability criterion, and the resultant layer is close to the threshold for which decoupling might be expected. The relative importance of CTEI versus decoupling to the sensitivity of this case is not, however, addressed in this study.

The first two conclusions undermine some of the classic arguments used to rationalize large-eddy simulation. At least for this case, the global behavior of the solutions depends on the most poorly understood details of the models, namely its numerics and the behavior of SGS models in regions of sharp gradients and phase changes. Such a situation challenges the idea (hope) that the proper rate of entrainment can be maintained if the energetics of the large eddies are well represented-if only because the representation of the small eddies may in the end mediate what component of the radiative forcing is actually felt by the large-scale (e.g., Lewellen and Lewellen 1998). Although the particulars of the observed case may make it a particularly challenging test for LES, such cases, which are on a knife edge between solid or broken cloud regimes, are also those most susceptible to perturbation and thus most desirable to quantify. This case is both challenging and extraordinarily well observed. It should serve as a useful benchmark for simulations with ever-increasing resolution, fueled by the importance of the topic and the ceaseless march embodied in Moore's law.

Acknowledgments. This case is the eighth case study conducted under the auspices of the GEWEX cloud systems studies (GCSS) boundary layer cloud working group. The support of the GCSS community continues to be instrumental in making this type of work possible.
The first author's research was supported by the NSF through Grant ATM-0097053. Bretherton and Zhu were supported by NASA Grant NAGS5-10624. The work of Golaz was performed while holding a National Research Council Research Associateship Award at the Naval Research Laboratory, Monterey, California. COAMPS is a registered trademark of the Naval Research Laboratory. Lewellen was supported in part by Grant N00014-98-1-0595 from the Office of Naval Research. The Dutch National Computing Facilities Foundation (NCF) sponsored Stephan de Roode for the use of supercomputer facilities. Martin Köhler is thanked for his comments on an early version of this manuscript. The advection schemes used by the UCLA-LES were developed from templates written by Verica SavicJovcic as part of the 2003 Institute for Pure and Applied Mathematics (IPAM) summer school on modern applied mathematics in the atmospheric and oceanic sciences. Ms. Savic-Jovcic is also thanked for her comments on an early draft of this manuscript.

\section{APPENDIX A Model Descriptions}

Many models share some features. For instance, all solve equations for the three components of velocity, mass fraction of total water (either total water specific humidity or mixing ratio), and liquid water potential temperature in three dimensions on an Arakawa $\mathrm{C}$ grid. The equations governing these fields are solved on a three-dimensional mesh whose horizontal discretization is over a $96^{2}$-point regular lattice, with 35 -m spacing and cyclic boundary conditions. Because the NCAR model uses a pseudospectral representation and explicit horizontal filtering, this representation yields an effective resolution of $52.5 \mathrm{~m}$. All except the Naval Research Laboratory (NRL) and Colorado State University (CSU) models (COAMPS and RAMS, respectively) invert Poisson equations to maintain continuity, and these inversions are uniformly based on fast Fourier transforms in the horizontal and an inversion of a tridiagonal matrix (in Fourier space) in the vertical. Most solve a thermodynamic equation that assumes that state variables are uniform within a grid volume. Hence the entire grid volume is either saturated or unsaturated; the one exception is the WVU model, which estimates the variance of thermodynamic quantities within a grid volume to estimate fractional cloudiness. The major differences among the models is in how they discretize the equations, how they model unresolved processes, and how pressure and density are decoupled from one another. These, along with the different configurations of the various models used for the purposes of this intercomparison, are detailed below. 
- DHARMA. DHARMA solutions were provided by A. Ackerman, M. Kirkpatrick, and D. Stevens. This model solves the Ogura-Phillips anelastic equations. The vertical discretization has a nominal spacing of $12 \mathrm{~m}$, which is reduced to $4 \mathrm{~m}$ at the surface, varying upward as the square of a sinusoid with an amplitude of $10 \mathrm{~m}$, back to $\delta z=4 \mathrm{~m}$ at the initial inversion height, and stretched once again above. The top of the domain is at $1500 \mathrm{~m}$, and a sponge layer occupies the upper $250 \mathrm{~m}$. The DHARMA-0 integration models the SGS fluxes using a dynamic SmagorinskyLilly model that computes local values of $C_{s}$ and $\operatorname{Pr}_{t}$ from the instantaneous flow conditions (Germano et al. 1991). With the dynamic SGS model, a modified version of the Brown et al. (2001) model is used to treat surface layer stresses in the bottom $2 \Delta x=70 \mathrm{~m}$ of the domain. The subgrid model is treated as an explicit source term. The DHARMA-1 configuration uses the static Smagorinsky-Lilly model with $c_{s}=$ $0.18, \delta=(\Delta x \Delta y \Delta z)^{1 / 3}$, a turbulent Prandtl number $\left(\operatorname{Pr}_{t}\right)$ of 0.40 . For both the dynamic and Smagorinsky model the stability on the grid scale is computed using a Richardson number following Mac Vean and Mason (1990). For all runs its equations are integrated using a forward-in-time method based on a second-order Runge-Kutta scheme with a thirdorder, total variation diminishing (TVD) upwinding advection scheme as described by Stevens and Bretherton (1996). The code is parallelized using the message passing interface with a $2 \mathrm{D}$ decomposition. The base calculations are performed on a 96-96-128 point mesh with a nominal time step of $2 \mathrm{~s}$. On sixteen 2.4-GHz Pentium-4 processors of a Beowulf cluster, one time step takes about $5 \mathrm{~s}$. Except for the dynamic SGS model, the model has been previously described by Stevens et al. (2002).

- IMAU. Institute for Marine and Atmospheric Research (IMAU) solutions were provide by S. de Roode. This LES model (vanZanten et al. 1999) is used by research groups at the IMAU (Utrecht University), the Royal Netherlands Meteorological Institute (KNMI), Delft University of Technology, and Wageningen University. It solves the Boussinesq equations using finite differences on a Cartesian grid with uniform a 5-m vertical grid that is translated with the mean geostrophic wind. A sponge layer is in effect for the upper 15 points. The advection of scalars is performed using the $\kappa=1 / 3$ TVD scheme. The leapfrog method with an Asselin filter is used to integrate in time. The SGS model is formulated following Deardorff (1980b). The code is parallelized using the message passing interface. To satisfy the Courant-Friedrichs-Lewy (CFL) criterion during the spinup phase the time step is $0.1 \mathrm{~s}$. With 16 processors on an SGI Origin 3800 one time step takes about $2.6 \mathrm{~s}$. - MPI. MPI solutions were provided by A. Chlond and F. Müller. The MPI-LES model solves the Boussinesq equations on a regular Cartesian grid with a 5 -m vertical spacing that is translated with the geostrophic wind to allow for larger time steps. A Rayleigh damping layer in the upper sixtieth of the domain was utilized to absorb vertically propagating gravity waves. The model includes most of the physical processes occurring in the moist PBL. The SGS model is based on a transport equation for the SGS turbulent energy following Deardorff (1980b), where moist processes are accounted for in the definition of the Richardson number for the MPI-0 and neglected for the MPI-1 configuration. Microphysical processes are modeled following Lüpkes' three-variable model. Advection of momentum is formulated using a second-order scheme that conserves the integral of linear and quadratic quantities up to very small errors. In the MPI- 0 configuration scalars are advected using the monotone scheme described by Chlond (1994), while for MPI-1 scalars are advected using the positive definite method described by Bott (1989). The time integration scheme uses a combination of second-order Adams-Bashforth and Euler steps. A time step of $0.8 \mathrm{~s}$ was used for all runs. The calculations are performed on eight processors of a NEC-SX6; one time step takes about $2.2 \mathrm{~s}$. Further information can be found in Chlond $(1992,1998)$.

- NCAR. NCAR solutions were provided by C-H. Moeng. The NCAR code solves the Boussinesq equations using a Fourier representation in the horizontal planes and finite differences in the vertical. The vertical mesh has uniform $5-\mathrm{m}$ spacing topped by a rigid lid after 400 points; no sponge layer is applied. Subgrid turbulent fluxes are modeled using the Deardorff's TKE model. All equations are time stepped using a third-order Runge-Kutta scheme with a variable time step that maintains maximum Courant numbers of about 0.5 . For the vertical advection terms, a second-order finite differencing is used for the momentum equations, while the $\kappa=1 / 3$ TVD scheme is used for the scalar equations. For the horizontal advection terms, one simulation NCAR-0 configuration uses pseudospectral differences, while for the NCAR-1 configuration the $\kappa=1 / 3$ scheme is applied in all directions. The code is parallelized using the message passing interface with a $1 \mathrm{D}(\mathrm{z})$ decomposition. On 80 processors (eight nodes and 10 tasks) of NCAR's Bluesky (IBM-SP), one time step takes about $4.7 \mathrm{~s}$.

- COAMPS. COAMPS solutions were provided by J.-C. Golaz. The COAMPS model is based on the 
Navy's Coupled Ocean/Atmosphere Mesoscale Prediction System (COAMPS; Hodur 1997). It solves the compressible equations of motion following Klemp and Wilhelmson (1978). Unlike the other models, its predictive scalars are $\theta$ and both $q_{v}$ and $q_{c}$. The vertical grid spacing is $25 \mathrm{~m}$ from the surface up to $390 \mathrm{~m}$, refined (5\% per layer) until a minimum value of $5 \mathrm{~m}$ and kept constant at $5 \mathrm{~m}$ up to $920 \mathrm{~m}$. Above this level, the grid spacing is stretched (5\% per layer) up to a maximum of $25 \mathrm{~m}$. The top of the domain is located at $1320 \mathrm{~m}$, and a sponge layer with a damping time of $60 \mathrm{~s}$ is applied to the seven uppermost model levels. For the COAMPS-1 configuration SGS fluxes are computed following Smagorinsky-Lilly, while for COAMPS-0 they are computed following Deardorff (1980b). The time-stepping scheme is leapfrog for the momentum variables with a Robert filter applied, and forward in time for the scalars. Second-order advection is used for the momentum variables and the second-order Bott (1989) scheme is used for the scalars. The code is parallelized using the message passing interface with a $2 \mathrm{D}$ domain decomposition. The main time step is $0.5 \mathrm{~s}$ with 16 nested acoustic time steps. The speed of the sound waves is reduced to 240 $\mathrm{m} \mathrm{s}^{-1}$. On a 20-processor SGI Origin 2000, one time step takes approximately $10 \mathrm{~s}$.

- RAMS. RAMS solutions were provided by H.-L. Jiang. The Colorado State RAMS model (Cotton et al. 2001) is constructed around the full set of nonhydrostatic, compressible equations. The vertical grid is $10 \mathrm{~m}$ except between 830 and $925 \mathrm{~m}$, where it is reduced to the requisite 5-m spacing. The top of the domain is at $1500 \mathrm{~m}$, and a sponge layer occupies the upper five levels. SGS fluxes are modeled following Deardorff (1980b). The momentum terms are time stepped using a hybrid combination of leapfrog and forward-in-time with an Asselin filter for the damping of the computational mode; momentum advection is computed using second-order centered differences. Scalar terms are time stepped using a forward scheme staggered with respect to the time levels of the momentum terms, so that the advecting winds correspond to the midpoint times. Scalar advection maintains the sign of the advected variable but is not monotonic. The base calculations are performed on a $96 \times 96 \times 160$ point mesh with a time step of $0.5 \mathrm{~s}$ on a single-processor $1.8-\mathrm{GHz}$ Linux machine.

- UCLA. UCLA solutions were provided by B. Stevens and J. Edwards. The UCLA model solves the Ogura-Phillips anelastic equations using finite differences on a regular-horizontal, stretched-vertical mesh. The vertical spacing is $10 \mathrm{~m}$ near the surface and refined (10\% per layer) to obtain a fixed 5-m (or less in the case of some sensitivity studies) spacing spanning the interval between 825 and $925 \mathrm{~m}$, above which the grid is stretched again. The top of the domain is at $1470 \mathrm{~m}$, and a sponge layer occupies the upper five levels. Subgrid fluxes are modeled using the Smagorinsky-Lilly model. The momentum terms are time stepped using a leapfrog scheme with an Asselin filter for the damping of the computational mode; momentum advection is computed using fourth-order centered differences. Scalar terms are time stepped using a forward scheme staggered with respect to the time levels of the momentum terms, so that the advecting winds correspond to the midpoint times. Scalar advection is TVD and uses the monotonized centered (MC) flux limiters. The code is parallelized using the message passing interface with a $1 \mathrm{D}$ decomposition. The base calculations are performed on a 96-96-131 point mesh with a time step of $0.667 \mathrm{~s}$; on eight processors of an IBM-SP4, one time step takes about $0.5 \mathrm{~s}$.

- SAM. The Colorado State University System for Atmospheric Modeling (SAM) solutions were provided by M. Khairoutdinov, C. Bretherton, and P. Zhu. SAM (Khairoutdinov and Randall 2003) solves the Ogura-Phillips anelastic equations on a Cartesian grid with a uniform 5-m vertical spacing and $35-\mathrm{m}$ horizontal spacing. The top of the domain is at $1600 \mathrm{~m}$, and a sponge layer occupies the upper $25 \%$ of the domain. The Deardorff (1980b) SGS model is used with $C_{s}=0.19(=0.54$ at the lowest grid level); $\mathrm{Pr}_{t}=0.33$ for neutral and unstable conditions. Given the anisotropy of the grid the SGS length scale is set to $\delta=\Delta z$ and the horizontal eddy-diffusion coefficients are computed by multiplying the vertical coefficients by a square of the ratio of horizontal to vertical grid spacings. The momentum equations are integrated second-order centered differences using the third-order Adams-Bashforth scheme with a variable time step. All the scalars are transported using a monotonic scheme. The code is parallelized using the message passing interface with a 2D domain decomposition. The base calculations are performed on a 96 $\times 96 \times 320$ point mesh with a time step of $1.0 \mathrm{~s}$ on eight processors of $350-\mathrm{MHz}$ SGI Origin workstation. One time step takes about $2.5 \mathrm{~s}$.

- METO. METO solutions were provided by E. Whelan and A. Lock. The Met Office model, as per Brown et al. (2002), solves Boussinesq equations (instead of anelastic) using an Arakawa $\mathrm{C}$ grid in the horizontal and a Lorenz grid in the vertical with variable vertical resolution. The nominal vertical spacing is $10 \mathrm{~m}$ refined to obtain a fixed $5-\mathrm{m}$ spacing spanning the interval between 700 and $900 \mathrm{~m}$ above which the 
grid is stretched again. The momentum terms are time stepped using a leapfrog scheme with a RobertAsselin time filter. Momentum advection is computed using the Piascek-Williams advection scheme. Scalar terms are time stepped using a leapfrog scheme with a Robert-Asselin time filter. Scalar advection is computed using the TVD/ULTIMATE scheme. The subgrid model follows the method of Smagorinsky-Lilly and is solved explicitly with the Richardson number in the stability dependence calculated using the method of MacVean and Mason (1990). The code is parallelized using general communication (GCOM) library routines with a 1D decomposition. The base calculations are performed on a 96-96-110 point mesh with a variable time step based on a Courant number of 0.4 using horizontal Gallilean transformation to minimize the Courant number. This gave an average time step of $0.125 \mathrm{~s}$. The model was run on four processors of an NEC SX-6, with one time step taking about $0.5 \mathrm{~s}$

- WVU. WVU solutions were provided by D. Lewellen. This model solves Boussinesq equations approximation using finite differences over an Arakawa C grid with stretched vertical spacing. The stretching rate within the layer is always $10 \%$ or less. There is damping imposed on the upper five levels. The subgrid model is a TKE scheme, with the subgrid turbulence length scale dependent on local grid spacing, distance from the surface, and Richardson number. A saturation variance is carried to implement subgrid partial cloudiness following Sommeria, Deardorff, and Mellor. The momentum terms (and TKE and saturation variance) are advanced using a leapfrog scheme in time and second-order centered differences in space (Piacsek and Williams 1970). Temperature and humidity are advected using the piecewise parabolic method ( $\mathrm{ppm})$. Diffusion terms are implemented implicitly in the vertical and explicitly in the horizontal (some with the DuFort-Frankel scheme). The time step is allowed to vary and the Courant condition is checked everywhere at each time step. The base case was run on a $96 \times 96 \times 118$ point grid with the time step $=0.5 \mathrm{~s}$ over most of the run. On an old single-processor Alpha Linux box, each time step took 19 s. WVU-0 differs from WVU-1 in that it uses the unsaturated Richardson number at cloud top when calculating SGS fluxes.

\section{APPENDIX B}

\section{Output Templates and Statistics}

Tables B1 and B2 show the templates for the output NetCDF files.
TABLE B1. Temporal statistics from LES. Each field is valid at a particular time.

\begin{tabular}{|c|c|c|}
\hline $\begin{array}{l}\text { NetCDF } \\
\text { short name }\end{array}$ & Description & Units \\
\hline Time & Time of sample & $\mathrm{s}$ \\
\hline zi_bar & $\begin{array}{l}\text { Average inversion height } \\
\quad\left(q_{t}=8 \mathrm{~g} \mathrm{~kg}^{-1} \text { is isoline }\right)\end{array}$ & $\mathrm{m}$ \\
\hline zi_var & Variance of inversion height & $\mathrm{m}^{2}$ \\
\hline zb_bar & Average cloud-base height & $\mathrm{m}$ \\
\hline zb_var & Variance of cloud-base height & $\mathrm{m}^{2}$ \\
\hline cfrac & Fraction of columns with condensate & $\%$ \\
\hline lwp_bar & Domain-averaged liquid water path & $\mathrm{g} \mathrm{m}^{-2}$ \\
\hline lwp_var & $\begin{array}{l}\text { Variance of liquid water path across } \\
\text { domain }\end{array}$ & $\mathrm{g}^{-2} \mathrm{~m}^{-4}$ \\
\hline tke & $\begin{array}{l}\text { Vertically averaged TKE (resolved } \\
\text { and subfilter) }\end{array}$ & $\mathrm{m}^{3} \mathrm{~m}^{-2}$ \\
\hline w2 & $\begin{array}{l}\text { Maximum value of layer-averaged } \\
\text { vertical velocity }\end{array}$ & $\mathrm{m}^{2} \mathrm{~s}^{-2}$ \\
\hline wstar & Convective velocity scale & $\mathrm{m} \mathrm{s}^{-1}$ \\
\hline lhf_bar & Domain-averaged latent heat flux & $\mathrm{W} \mathrm{m}^{-2}$ \\
\hline shf_bar & Domain-averaged sensible heat flux & $\mathrm{W} \mathrm{m}^{-2}$ \\
\hline ustar & Surface friction velocity & $\mathrm{K}^{2}$ \\
\hline
\end{tabular}

TABLE B2. Profile statistics from LES. Excepting the time and height fields, which do not vary with time, each field consists of eight vertical profiles averaged over 30-min intervals and one initial profile. The vertical profiles are either given at temperature or $w$ points depending on the field.

\begin{tabular}{|c|c|c|}
\hline $\begin{array}{l}\text { NetCDF } \\
\text { short name }\end{array}$ & Description & Units \\
\hline Time & End of averaging interval & $\mathrm{s}$ \\
\hline $\mathrm{zt}$ & Height of temperature points & $\mathrm{m}$ \\
\hline zW & Height of $w$ points & $\mathrm{m}$ \\
\hline$u$ & Zonal wind speed & $\mathrm{m} \mathrm{s}^{-1}$ \\
\hline$v$ & Meridional wind speed & $\mathrm{m} \mathrm{s}^{-1}$ \\
\hline theta_1 & Liquid-water potential temperature & $\mathrm{K}$ \\
\hline $\mathrm{rt}-$ & Total water mixing ratio & $\mathrm{g} \mathrm{kg}^{-1}$ \\
\hline $\mathrm{rl}$ & Liquid-water mixing ratio & $\mathrm{g} \mathrm{kg}^{-1}$ \\
\hline $\operatorname{dn} 0$ & Density & $\mathrm{kg} \mathrm{m}^{-3}$ \\
\hline u_var & Resolved variance of zonal wind & $\mathrm{m}^{2} \mathrm{~s}^{-2}$ \\
\hline v_var & Resolved variance of meridional wind & $\mathrm{m}^{2} \mathrm{~s}^{-2}$ \\
\hline w_var & Resolved variance of vertical wind & $\mathrm{m}^{2} \mathrm{~s}^{-2}$ \\
\hline w_skw & Resolved third moment of vertical wind & $\mathrm{m}^{2} \mathrm{~s}^{-2}$ \\
\hline $\mathrm{e}$ & Subfilter turbulence kinetic energy & $\mathrm{m}^{2} \mathrm{~s}^{-2}$ \\
\hline theta_var & Resolved variance of $\theta_{l}$ & $\mathrm{~K}^{2}$ \\
\hline rt_var & Resolved variance of $r_{t}$ & $\mathrm{~g}^{2} \mathrm{~kg}^{-2}$ \\
\hline rl_var & Resolved variance of $r_{t}$ & $\mathrm{~g}^{2} \mathrm{~kg}^{-2}$ \\
\hline rad_flx & Radiative flux & $\mathrm{W} \mathrm{m}{ }^{-2}$ \\
\hline sfs_tw & Modeled subfilter $\theta_{l}$ flux & $\mathrm{W} \mathrm{m}^{-2}$ \\
\hline tot_tw & Resolved $\theta_{l}$ flux & $\mathrm{W} \mathrm{m}^{-2}$ \\
\hline sfs_rw & Modeled subfilter $r_{t}$ flux & $\mathrm{W} \mathrm{m}^{-2}$ \\
\hline tot_rw & Resolved $r_{t}$ flux & $\mathrm{W} \mathrm{m}^{-2}$ \\
\hline sfs_uw & Modeled subfilter $u$ flux & $\mathrm{m}^{2} \mathrm{~s}^{-2}$ \\
\hline tot_uw & Resolved $u$ flux & $\mathrm{m}^{2} \mathrm{~s}^{-2}$ \\
\hline sfs_vw & Modeled subfilter $\nu$ flux & $\mathrm{m}^{2} \mathrm{~s}^{-2}$ \\
\hline tot_vw & Resolved $\nu$ flux & $\mathrm{m}^{2} \mathrm{~s}^{-2}$ \\
\hline E & Resolved turbulence kinetic energy & $\mathrm{m}^{2} \mathrm{~s}^{-2}$ \\
\hline shr_prd & Shear production of $E$ & $\mathrm{~m}^{2} \mathrm{~s}^{-3}$ \\
\hline boy_prd & Buoyancy production of $E$ & $\mathrm{~m}^{2} \mathrm{~s}^{-3}$ \\
\hline Transport & Resolved transport of $E$ & $\mathrm{~m}^{2} \mathrm{~s}^{-3}$ \\
\hline Dissipation & Dissipation of $E$ & $\mathrm{~m}^{2} \mathrm{~s}^{-3}$ \\
\hline Storage & Change in $E$ over averaging interval & $\mathrm{m}^{2} \mathrm{~s}^{-3}$ \\
\hline
\end{tabular}



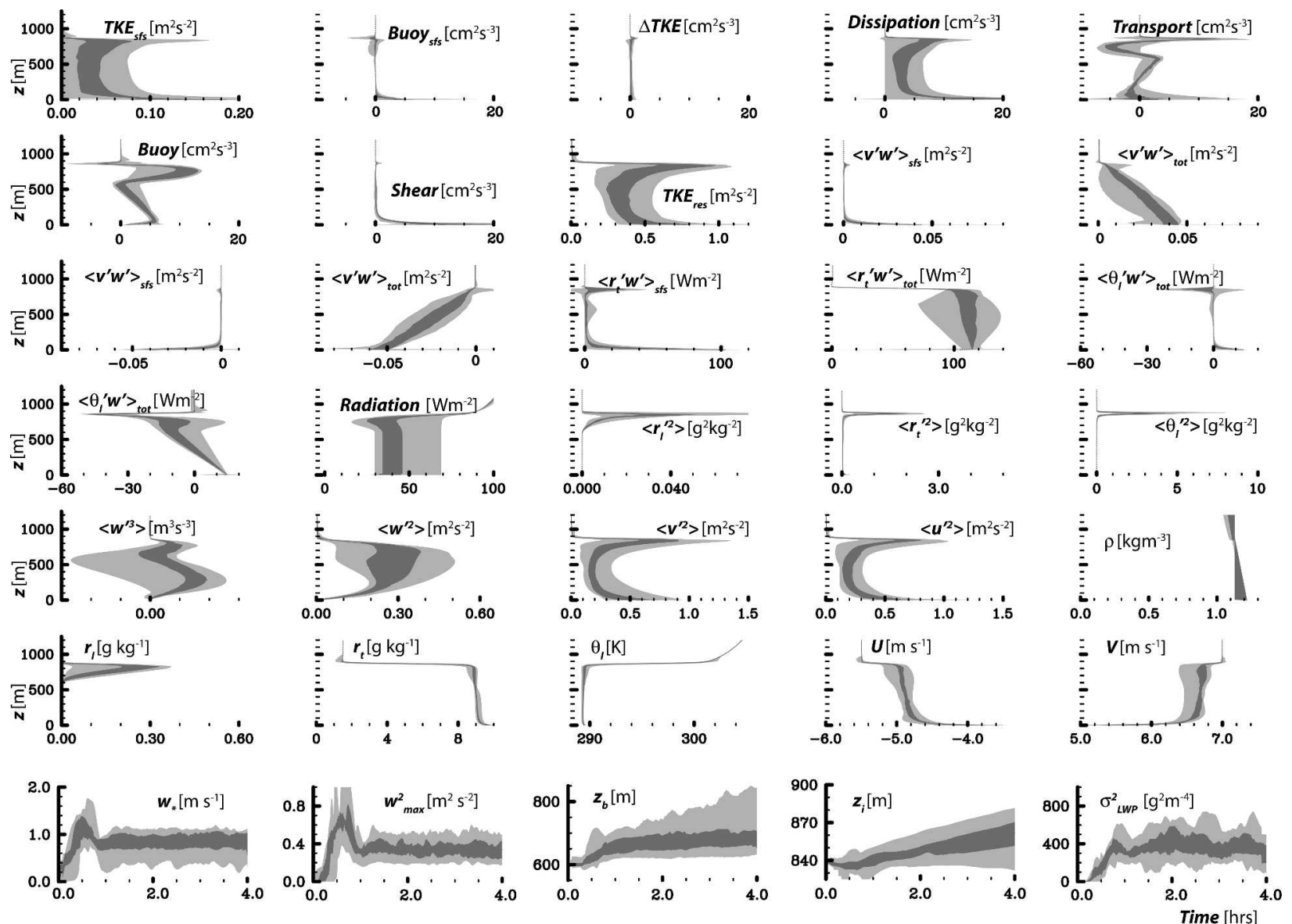

FIG. C1. (upper six rows) Thumbnails of profile and (last row) time series statistics for the master ensemble.

\section{APPENDIX C}

\section{Thumbnail}

Figure $\mathrm{C} 1$ gives an overview of the model profile statistics.

\section{REFERENCES}

Albrecht, B. A., D. A. Randall, and S. Nicholls, 1988: Observations of marine stratocumulus clouds during FIRE. Bull. Amer. Meteor. Soc., 69, 618-626.

Bott, A., 1989: A positive definite advection scheme obtained by nonlinear renormalization of the advective fluxes. Mon. Wea. Rev., 117, 1006-1015.

Bradshaw, P., 1972: The understanding and prediction of turbulent flow. Aeronaut. J., 76, 403-418.

Bretherton, C. S., and M. C. Wyant, 1997: Moisture transport, lower tropospheric stability, and decoupling of cloud-topped boundary layers. J. Atmos. Sci., 54, 148-167.

, and Coauthors, 1999: An intercomparison of radiativelydriven entrainment and turbulence in a smoke cloud, as simulated by different numerical models. Quart. J. Roy. Meteor. Soc., 125, 391-423.

Brown, A., J. M. Hobson, and N. Wood, 2001: Large-eddy simu- lation of neutral turbulent flow over rough sinusoidal ridges. Bound.-Layer Meteor., 98, 411-441.

_ and Coauthors, 2002: Large-eddy simulation of the diurnal cycle of shallow cumulus convection over land. Quart. J. Roy. Meteor. Soc., 128, 1779-1798.

Chlond, A., 1992: Three-dimensional simulation of cloud street development during a cold air outbreak. Bound.-Layer Meteor., 58, 161-200.

- 1994: Locally modified version of Bott's advection scheme. Mon. Wea. Rev., 122, 111-125.

, 1998: Large-eddy simulation of contrails. J. Atmos. Sci., 55, 796-819.

Cotton, W. R., R. Pielke, R. Walko, G. Liston, C. Tremback, H. Jiang, J. Y. Harrington, and G. Feingold, 2001: RAMS 2001: Current status and future directions. Meteor. Atmos. Phys., 82, 5-29.

Deardorff, J. W., 1980a: Cloud top entrainment instability. J. Atmos. Sci., 37, 131-147.

_ 1980b: Stratocumulus-capped mixed layers derived from a three-dimensional model. Bound.-Layer Meteor., 18, 495527.

Duynkerke, P. G., and Coauthors, 2004: Observations and numerical simulations of the diurnal cycle of the EUROCS stratocumulus case. Quart. J. Roy. Meteor. Soc., 130, 3269-3296.

Faloona, I., and Coauthors, 2005: Observations of entrainment in 
eastern Pacific marine stratocumulus using three conserved scalars. J. Atmos. Sci., in press.

$\mathrm{Fu}$, Q., and K.-N. Liou, 1993: Parameterization of the radiative properties of cirrus clouds. J. Atmos. Sci., 50, 2008-2025.

Gerber, H., G. Frick, S. Malinowski, J.-L. Brenguier, and F. Burnet, 2005: Holes and entrainment in stratocumulus. J. Atmos. Sci., 62, 443-459.

Germano, M., U. Piomelli, P. Moin, and W. H. Cabot, 1991: A dynamic subgrid-scale eddy viscosity model. Phys. Fluids, A3, $1760-1765$.

Ghosal, S., 1996: An analysis of numerical errors in large-eddy simulations of turbulence. J. Comput. Phys., 125, 187-206.

Hodur, R. M., 1997: The Naval Research Laboratory's Coupled Ocean/Atmosphere Mesoscale Prediction System (COAMPS). Mon. Wea. Rev., 125, 1414-1430.

Khairoutdinov, M. F., and D. A. Randall, 2003: Cloud resolving modeling of the ARM summer 1997 IOP: Model formulation, results, uncertainties and sensitivities. J. Atmos. Sci., 60, 607625 .

Klemp, J. B., and R. B. Wilhelmson, 1978: The simulation of three-dimensional convective storm dynamics. J. Atmos. Sci., 35, 1070-1095.

Lenschow, D. H., 1996: A proposal for measuring entrainment into the cloud-capped boundary layer. Proc. ETL/CSU Cloud-Related Process Modeling and Measurement Workshop, Boulder, CO, NOAA/ETL, 29-55.

Lewellen, D. C., and W. Lewellen, 1998: Large-eddy boundary layer entrainment. J. Atmos. Sci., 55, 2645-2665.

—, and —, 2002: Entrainment and decoupling relations for cloudy boundary layers. J. Atmos. Sci., 59, 2966-2986.

Lilly, D. K., 1968: Models of cloud topped mixed layers under a strong inversion. Quart. J. Roy. Meteor. Soc., 94, 292-309.

Lock, A. P., 1998: The parameterization of entrainment in cloudy boundary layers. Quart. J. Roy. Meteor. Soc., 124, 2729-2753.

MacVean, M. K., and P. J. Mason, 1990: Cloud-top entrainment instability through small-scale mixing and its parameterization in numerical models. J. Atmos. Sci., 47, 1012-1030.

Moeng, C.-H., 2000: Entrainment rate, cloud fraction and liquid water path of PBL stratocumulus clouds. J. Atmos. Sci., 57, $3627-3643$.

, and Coauthors, 1996: Simulation of a stratocumulus-topped PBL: Intercomparison among different numerical codes. Bull. Amer. Meteor. Soc., 77, 261-278.

, B. Stevens, and P. S. Sullivan, 2005: Where is the interface of the stratocumulus-topped PBL? J. Atmos. Sci., in press.
Piacsek, S. A., and G. P. Williams, 1970: Conservation properties of convection difference schemes. J. Comput. Phys., 6, 392405.

Randall, D. A., 1980: Conditional instability of the first kind upside-down. J. Atmos. Sci., 37, 125-130.

Stevens, B., 2000a: Cloud transitions and decoupling in shear-free stratocumulus-topped boundary layers. Geophys. Res. Lett., 27, 2557-2560.

_ 2000b: Quasi-steady analysis of a PBL model with an eddydiffusivity profile and nonlocal fluxes. Mon. Wea. Rev., 128, 824-836.

_ 2002: Entrainment in stratocumulus mixed layers. Quart. J. Roy. Meteor. Soc., 128, 2663-2690.

_ C.-H. Moeng, and P. P. Sullivan, 1999: Large-eddy simulations of radiatively driven convection: Sensitivities to the representation of small scales. J. Atmos. Sci., 56, 3963-3984.

— der a strong inversion. J. Atmos. Sci., 58, 1870-1891.

- , and Coauthors, 2003a: On entrainment in nocturnal marine stratocumulus. Quart. J. Roy. Meteor. Soc., 129, 3469-3492.

— , and Coauthors, 2003b: DYCOMS-II. Bull. Amer. Meteor. Soc., 84, 579-593.

Stevens, D., and C. S. Bretherton, 1996: A new forward-in-time advection scheme and adaptive multilevel flow solver for nearly incompressible atmospheric flow. J. Comput. Phys., 129, 284-295.

— A. S. Ackerman, and C. S. Bretherton, 2002: Effect of domain size and numerical resolution on the simulation of shallow cumulus convection. J. Atmos. Sci., 59, 3285-3301.

Turton, J. D., and S. Nicholls, 1987: A study of the diurnal variation of stratocumulus using a multiple mixed layer model. Quart. J. Roy. Meteor. Soc., 113, 969-1009.

vanZanten, M. C., P. G. Duynkerke, and J. W. M. Cuijpers, 1999: Entrainment parameterization in convective boundary layers derived from large eddy simulations. J. Atmos. Sci., 56, 813828.

Wyngaard, J. C., 1998: Experiment, numerical modeling, numerical simulation, and their roles in the study of convection. Buoyant Convection in Geophysical Flows, Vol. 513, E. J. Plate et al., Eds., Kluwer Academic, 239-251.

Zhu, P., and Coauthors, 2005: Intercomparison and interpretation of single-column model simulations of a nocturnal stratocumulus-topped marine boundary layer. Mon. Wea. Rev., in press. 